\title{
Surface density function evolution and the influence of strain rates during turbulent boundary layer flashback of hydrogen-rich premixed combustion
}

\section{$\operatorname{AUTHOR}(S):$}

Ahmed, Umair; Pillai, Abhishek L.; Chakraborty, Nilanjan; Kurose, Ryoichi

\section{CITATION:}

Ahmed, Umair ... [et al]. Surface density function evolution and the influence of strain rates during turbulent boundary layer flashback of hydrogen-rich premixed combustion.

Physics of Fluids 2020, 32(5): 055112.

\section{ISSUE DATE:}

\section{0-05-29}

URL:

http://hdl.handle.net/2433/254488

\section{RIGHT:}

This article may be downloaded for personal use only. Any other use requires prior permission of the author and AIP Publishing. This article appeared in Phys. Fluids 32, 055112 (2020); and may be found at https://doi.org/10.1063/5.0004850.; The full-text file will be made open to the public on 29 May 2021 in accordance with publisher's 'Terms and Conditions for Self-Archiving'. 


\section{Surface density function evolution and the influence of strain rates during turbulent boundary layer flashback of hydrogen-rich premixed combustion}

Cite as: Phys. Fluids 32, 055112 (2020); https://doi.org/10.1063/5.0004850

Submitted: 15 February 2020 . Accepted: 04 May 2020 . Published Online: 29 May 2020

Umair Ahmed (D), Abhishek L. Pillai, Nilanjan Chakraborty (D), and Ryoichi Kurose
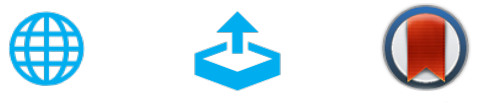

View Online

Export Citation

CrossMark

\section{ARTICLES YOU MAY BE INTERESTED IN}

Wake topology and dynamics over a slender body at a high incidence and their relation to structural loading

Physics of Fluids 32, 055111 (2020); https://doi.org/10.1063/5.0008952

Improving the $k-\omega-\gamma-A_{r}$ transition model by the field inversion and machine learning framework

Physics of Fluids 32, 064101 (2020); https://doi.org/10.1063/5.0008493

Non-linear instability analysis of the three-dimensional Navier-Stokes equations: TaylorGreen vortex problem

Physics of Fluids 32, 064102 (2020); https://doi.org/10.1063/5.0006683

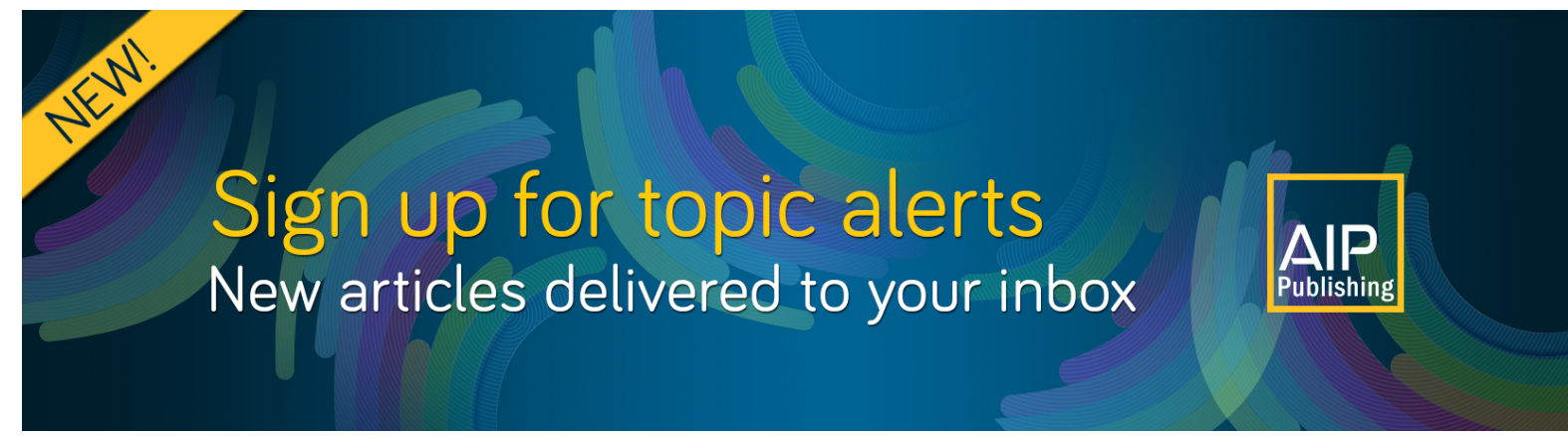




\title{
Surface density function evolution and the influence of strain rates during turbulent boundary layer flashback of hydrogen-rich premixed combustion
}

\author{
Cite as: Phys. Fluids 32, 055112 (2020); doi: 10.1063/5.0004850 \\ Submitted: 15 February 2020 - Accepted: 4 May 2020 • \\ Published Online: 29 May 2020

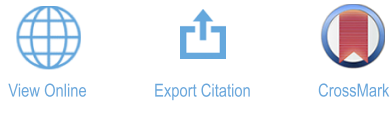

Umair Ahmed, ${ }^{1, a)}$ (D) Abhishek L. Pillai, ${ }^{2}$ Nilanjan Chakraborty, ${ }^{1}$ (D) and Ryoichi Kurose ${ }^{2}$

\begin{abstract}
AFFILIATIONS
1School of Engineering, Newcastle University, Newcastle upon Tyne NE1 7RU, United Kingdom

${ }^{2}$ Department of Mechanical Engineering and Science, Kyoto University, Kyoto daigaku-Katsura, Nishikyo-ku, Kyoto 615-8540, Japan
\end{abstract}

\begin{abstract}
a) Author to whom correspondence should be addressed: umair.ahmed@newcastle.ac.uk
\end{abstract}

\begin{abstract}
The statistical behavior of the magnitude of the reaction progress variable gradient [alternatively known as the surface density function (SDF)] and the strain rates, which govern the evolution of the SDF, have been analyzed for boundary layer flashback of a premixed hydrogen-air flame with an equivalence ratio of 1.5 in a fully developed turbulent channel flow. The non-reacting part of the channel flow is representative of the friction velocity based Reynolds number $R e_{\tau}=120$. A skeletal chemical mechanism with nine chemical species and twenty reactions is employed to represent hydrogen-air combustion. Three definitions of the reaction progress variable (RPV) based on the mass fractions of $\mathrm{H}_{2}, \mathrm{O}_{2}$, and $\mathrm{H}_{2} \mathrm{O}$ have been considered to analyze the SDF statistics. It is found that the mean variations of the SDF and the displacement speed $S_{d}$ depend on the choice of the RPV and the distance away from the wall. The preferential alignment of the RPV gradient with the most extensive principal strain rate strengthens with an increase in distance from the cold wall, which leads to changes in the behaviors of normal and tangential strain rates from the vicinity of the wall toward the middle of the channel. The differences in displacement speed statistics for different choices of the RPV and the wall distance affect the behaviors of the normal strain rate due to flame propagation and curvature stretch. The relative thickening/thinning of the reaction layers of the major species has been explained in terms of the statistics of the effective normal strain rate experienced by the progress variable isosurfaces for different wall distances and choices of RPVs.
\end{abstract}

Published under license by AIP Publishing. https://doi.org/10.1063/5.0004850

\section{INTRODUCTION}

Reduction in greenhouse gases and the control of pollutant emissions are becoming increasingly important for the power generation industry. Hydrogen-rich fuels are being considered as an alternative fuel for clean and efficient large-scale power generation. This mode of combustion offers a lower environmental impact and higher energy efficiency ${ }^{2}$ as hydrogen remains stable across a range of fuel concentrations during combustion and can be ignited with relative ease due to a high flammable range and high burning velocity. However, the aforementioned characteristics of hydrogen lead to a risk of flashback, which is an uncontrolled transient upstream propagation of a flame, and therefore make the development of hydrogen combustors much more difficult. ${ }^{2}$ Among the many challenges in enabling hydrogen-rich combustion for gas turbine applications, a fundamental understanding of flame flashback, especially for non-conventional and highly reactive hydrogen-rich fuels, remains an open question. ${ }^{3}$ The increased reactivity of hydrogen-rich syngas complicates the problem of boundary layer flashback considerably. Specifically, compared with hydrocarbon-air flames, hydrogen-air premixed flames are able to propagate three times (in relation to the flame thickness) closer to the wall before the heat loss to the solid surface leads to quenching, ${ }^{4}$ thus making it harder to understand the already complex 
physical and chemical phenomena ${ }^{5,6}$ involved in flame-wall interaction. When compared with their methane-air counterparts, hydrogen-air flames can propagate closer to the wall in regions of the boundary layer characterized by very low flow velocities. This also leads to increased heat transfer, which can potentially damage the combustor walls, thus consequently leading to a failure of the combustion equipment. Current modeling approaches [i.e., Reynolds averaged Navier-Stokes (RANS) or large eddy simulation (LES) techniques] used to simulate industrial scale combustors cannot accurately account for the unsteady flame dynamics involved in boundary layer flashback.

In turbulent premixed combustion, the unclosed mean/filtered reaction rate in the context of RANS and LES is usually closed using gradients of the reaction progress variable $(\mathrm{RPV}) c$ and relies on the generalized Flame Surface Density (FSD) ${ }^{7}$ or scalar dissipation rate $(\mathrm{SDR})^{8}$ modeling. Therefore, understanding of the statistical behavior of the modulus of the RPV gradient $|\nabla c|$, commonly referred to as the Surface Density Function (SDF), is of fundamental importance in the modeling of turbulent premixed combustion. ${ }^{9}$ Pope $^{10}$ and Candel and Poinsot ${ }^{11}$ demonstrated the role of the tangential strain rate and curvature on the evolution of $|\nabla c|$ by deriving a transport equation for the SDF. Direct Numerical Simulation (DNS) based analysis of Kollmann and Chen ${ }^{9}$ focused on the transport of $|\nabla c|$ and analyzed pocket formation in premixed flames. The strain rate and curvature dependence of the different terms of the SDF transport equation have been investigated for different turbulence conditions ${ }^{12,13}$ and for different fuels. ${ }^{14,15}$ The relative alignment of $\nabla c$ with the local principal strain rates has been investigated in several previous studies. ${ }^{13,16,17}$ These investigations found that $\nabla c$ preferentially aligns with the most extensive principal strain rate when the strain rate induced by heat release dominates over the straining induced by turbulent fluid motion, whereas a preferential alignment of $\nabla c$ with the most compressive principal strain rate is obtained when turbulent straining is stronger than the flame-induced strain rate. Recently, the influences of normal and tangential strain rates arising from flame propagation on the SDF evolution have been demonstrated ${ }^{18,19}$ and termed additional strain rates.

Most numerical investigations on the SDF transport and strain rate dependence of the SDF have been performed on canonical configurations (i.e., flame interacting with isotropic decaying turbulence). ${ }^{12-15,17-20}$ Recently, some attempts have been made to understand the influence of mean shear on the behavior of scalar gradients and the SDF in the case of Bunsen flames, ${ }^{21}$ turbulent jet flames under high Karlovitz numbers, ${ }^{22}$ and temporally evolving slot jet premixed flames. ${ }^{23}$ Sankaran et al. ${ }^{21}$ reported that the flame thickens in the mean sense, which is in contradiction to the earlier findings from a canonical configuration. ${ }^{20}$ It has been found that the statistical behavior of the strain rate induced by flame propagation in the case of high Karlovitz number jet flames ${ }^{22}$ is in contradiction with the earlier results obtained from flames interacting with decaying turbulence. ${ }^{18}$ The SDF statistics in the case of the turbulent bluff body burner have been found to be significantly affected by the downstream distance away from the bluff body due to variations in the shear rate in the axial direction. ${ }^{2}$

However, all of the aforementioned studies have been carried out for flames without the influence of the wall. Although fluiddynamic straining and strain rates arising from flame propagation are known to have influences on the SDF evolution, ${ }^{12-15,17-24}$ the effects of wall-induced straining on the SDF evolution in the turbulent boundary layer is yet to be analyzed in detail for wall-bounded turbulent flows. In the present work, we analyze the SDF evolution by interrogating a DNS database ${ }^{25,26}$ of turbulent boundary layer flashback. This database has previously been used to investigate the effects of pressure oscillations ${ }^{25}$ and turbulent kinetic energy transport physics ${ }^{26}$ during turbulent boundary layer flashback, but none of the previous analysis focused on the statistical behavior of the reactive scalar gradient evolution and the strain rates, which affect the SDF evolution. Understanding of the aforementioned statistics related to the SDF is necessary for improving $\mathrm{FSD}^{7}$ and $\mathrm{SDR}^{8}$ modeling. The main objectives of the present work are to understand the statistical behaviors of the different mechanisms, which control the evolution of the SDF in turbulent boundary layer flashback of hydrogen-rich premixed flames.

The paper is organized as follows. In Secs. II and III, the details for the DNS data and the mathematical background for the current analysis are provided. This is followed by the results in Sec. IV, and the conclusions are summarized in Sec.V.

\section{DIRECT NUMERICAL SIMULATION DATA}

The DNS data of boundary layer flashback performed by Kitano et $a .^{25}$ have been considered in this study. The flow configuration, turbulence, and flame characteristics are similar to the one used in the earlier work of Gruber et al. ${ }^{4}$ This database is representative of flashback in a channel flow at bulk Reynolds number $R e_{b}=\rho u_{b} 2 h / \mu=3500$, where $h$ is the channel half height and $u_{b}=1 / 2 h \int_{0}^{2 h} u d y=19.83 \mathrm{~m} / \mathrm{s}$, and the Reynolds number based on the channel half height and friction velocity $R e_{\tau}=\rho u_{\tau} h / \mu=120$, with $u_{\tau}=\sqrt{\left|\tau_{w}\right| / \rho}$ and $\tau_{w}=\mu \partial u /\left.\partial y\right|_{y=0 \text { or } y=2 h}$ being the friction velocity and wall shear stress, respectively. The simulation has been performed using the code known as $\mathrm{FK}^{3}$, which has been used in several previous studies on turbulent, reacting, and multiphase flows. ${ }^{27-31}$ The code solves conservation equations for mass, momentum, enthalpy, and chemical species in the context of the finite volume framework. A skeletal chemical mechanism comprising nine chemical species and twenty reactions ${ }^{32}$ is used to represent hydrogen-air combustion. The spatial derivatives for the momentum equation are evaluated via a fourth-order centered scheme. The convective terms of enthalpy and species mass fractions are calculated by using a third-order quadratic upstream interpolation for convective kinematics (QUICK) ${ }^{33}$ scheme. A second-order centered scheme is used to calculate all the other terms in the scalar transport equations. This way of solving the scalar equations is a common practice in many numerical investigations of heat transfer in wall bounded flows ${ }^{34,35}$ and ensures numerical stability of the code. The pressure-based semi-implicit algorithm for compressible flows proposed by Moureau et al. ${ }^{36}$ is used to solve the equations. In this algorithm, the governing equations are solved by using a fractionalstep method based on "characteristic splitting" to separate advection and acoustics (i.e., decoupling acoustics from advection). In this fractional-step algorithm, the first step (predictor step) consists of advecting the conserved variables, and the third-order explicit total variation diminishing (TVD) Runge-Kutta method is used for the time integration of the convective terms. Chemical kinetics are also solved and integrated using the multi-timescale (MTS) method in 
this step with a minimum time resolution of $1 \times 10^{-9} \mathrm{~s}$. The second step of this fractional-step algorithm is the pressure-correction step, wherein a Helmholtz equation is solved implicitly using the Stabilized Bi-Conjugate-Gradient (BiCGStab) solver. Hence, acoustic waves are solved implicitly. The multicomponent diffusion for different chemical species is evaluated via the diffusion velocities by solving the system of linear equations proposed in Refs. 37 and 38 for all grid points at all time steps and in all directions. The binary diffusion coefficients required for diffusion calculation are obtained from CHEMKIN. ${ }^{39}$ Further details on the numerical techniques used in this simulation can be found in the earlier work of Kitano et al. ${ }^{25}$

The computational domain for the DNS is divided into two regions, namely, the channel flow region and the buffer region, as shown in Fig. 1. The channel flow region is further sub-divided into two parts, namely, the turbulence generation region and the flashback region as described in Refs. 25 and 26. The dimensions of the channel flow region are $173 \mathrm{~mm} \times 20 \mathrm{~mm} \times 30 \mathrm{~mm}$, of which the flashback region consists of $103 \mathrm{~mm} \times 20 \mathrm{~mm} \times 30 \mathrm{~mm}$. In the turbulence generation region of the channel flow, a fully developed wall-bounded turbulent flow is generated by imposing a pressure drop and a periodic boundary condition in the $x$ direction. Further details on the non-reacting turbulence introduced into the flashback region of the channel can be found in Ref. 26. In the flashback region of the channel, the outflow characteristics of the upstream channel are introduced, and a freely propagating planar flame is initialized in the domain after $100 \mathrm{~ms}$ of the flow becoming fully turbulent in the channel. A no-slip isothermal boundary condition at $750 \mathrm{~K}$ is applied on the walls in the $y$ direction, while the $z$ direction is treated as periodic. The Navier-Stokes Characteristic Boundary Condition $(\mathrm{NSCBC})^{40}$ is applied at the outflow boundaries in the buffer region, as shown in Fig. 1.

The initial gas temperature, pressure, and equivalence ratio are $750 \mathrm{~K}, 0.1 \mathrm{MPa}$, and 1.5, respectively. The laminar burning velocity $S_{L}$ and the thermal flame thickness $\delta_{t h}=\left(T_{a d}-T_{R}\right) / \max |\nabla T|_{L}$ (where $T_{R}$ is the reactant temperature, $T_{a d}$ is the adiabatic flame temperature, and the subscript $L$ represents the laminar flame quantities) under these conditions are determined to be $14 \mathrm{~m} / \mathrm{s}$ and

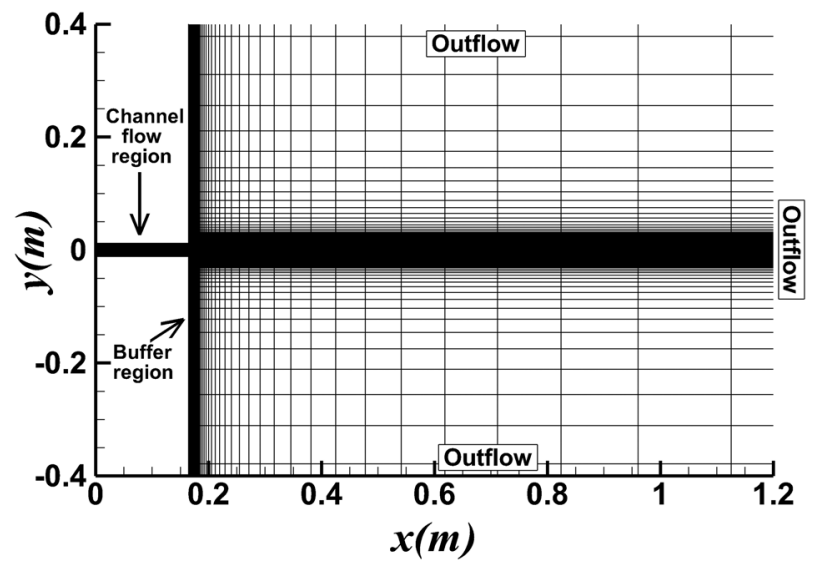

FIG. 1. Computational grid used for the direct numerical simulation shown on the $x-y$ mid-plane.
$0.48 \mathrm{~mm}$, respectively. ${ }^{41}$ The grid resolution in the flashback region of the simulation is $50 \mu \mathrm{m}$, which in non-dimensional wall units is $\Delta x^{+}=\Delta y^{+}=\Delta z^{+}=0.6$. This level of resolution is appropriate as it ensures that the laminar flame thermal thickness $\delta_{t h}$ is resolved in approximately 10 grid points and satisfies the resolution criteria for turbulent boundary layers, as recommended by Moser et al. ${ }^{42} \mathrm{~A}$ larger grid spacing of $700 \mu \mathrm{m}\left(\Delta x^{+}=8.4\right)$ is used in the $x$ direction of the turbulence generation region of the simulation as this level of resolution is sufficient to resolve the non-reacting turbulence at the conditions used in this work. A total of approximately $0.4 \times 10^{9}$ grid points are used in the simulation of which $1150 \times 400 \times 600$ are in the flashback region of the simulation.

In the post-processing of the data, the quantities of interest at a given value of the progress variable are ensemble averaged for 2.2 $\times 10^{-5} \mathrm{~s}$ and also in the periodic $(z)$ direction for a given value of $y / h$. The data are sampled after $1.6 \mathrm{~ms}$ from the time when the flame is initialized in the domain, which ensures that a steady flashback speed has been attained, and a further explanation of this is provided in the earlier work of Kitano et al. ${ }^{25}$ A similar technique has been used in the analysis of freely propagating statistically planner turbulent premixed flames ${ }^{7,43}$ and laboratory scale jet flames, ${ }^{21,22,24}$ and no area-weighting is carried out in the current analysis. Note that in the results presented below, only the region containing the flame (i.e., flashback region of the channel) is considered as the gradients of the chemical species remain zero outside this region during the simulation and will not affect the statistics presented in this work.

\section{MATHEMATICAL BACKGROUND}

The reactive flow field can be expressed in terms of RPV $c$, which can be defined as $c \equiv\left(Y_{R}-Y_{k}\right) /\left(Y_{R}-Y_{P}\right)$, where $Y_{k}$ represents the mass fraction of species $k$ (i.e., the species chosen to define the progress variable) and the subscripts $R$ and $P$ indicate the values of the species in the unburned and fully burned gases, respectively. Note that the progress variable derived from the nondimensional temperature is not valid in this case as the wall temperature is the same as that of the unburned gases, which leads to a non-dimensional temperature or the temperature based progress variable to be zero in the near wall region even though chemical activities remain strong close to the wall in the case of flashback. Moreover, local differential diffusion effects may lead to local occurrences of super-adiabatic temperature in $\mathrm{H}_{2}$-air flames, which gives rise to unphysical $c>1$ values. This makes it impossible to compare the SDF in the near wall region with the SDF toward the middle of the channel flow. The transport equation for $c$ is given by

$$
\rho \frac{\partial c}{\partial t}+\rho u_{j} \frac{\partial c}{\partial x_{j}}=\frac{\partial}{\partial x_{j}}\left(\rho D \frac{\partial c}{\partial x_{j}}\right)+\dot{\omega}_{c}
$$

where $\rho$ is the density, $u_{j}$ is the velocity component in the $j$-th direction, $D$ is the mass diffusivity of the progress variable, and $\dot{\omega}_{c}$ is the chemical reaction rate. The mass diffusivity of the progress variable in this case is evaluated as $D=\left(1-Y_{k}\right) /\left(\sum_{j \neq k} X_{j} / D_{j k}\right),{ }^{38}$ where $X_{j}$ is the mole fraction of species $j, D_{j k}$ is the binary diffusion coefficient, and species $k$ is used to define the reaction progress variable. Equation (1) can be expressed in the kinematic form for a given $c$ isosurface as

$$
\left(\frac{\partial c}{\partial t}+u_{j} \frac{\partial c}{\partial x_{j}}\right)=S_{d}|\nabla c|,
$$


where $S_{d}$ is the displacement speed and is defined as ${ }^{44,45}$

$$
S_{d}=\frac{\left(\dot{\omega}_{c}+\nabla \cdot(\rho D \nabla c)\right)}{(\rho|\nabla c|)} .
$$

It is evident that the displacement speed is affected by the reaction diffusion balance $\dot{\omega}_{c}+\nabla \cdot(\rho D \nabla c)$ and the SDF. As the displacement speed depends on the interplay between the reaction and the molecular diffusion rate, it is useful to express $S_{d}$ in terms of three different components as $S_{d}=S_{r}+S_{n}+S_{t}{ }^{45}$ The expressions for the reaction component $S_{r}$, normal diffusion component $S_{n}$, and the tangential diffusion contribution $S_{t}$ in displacement speed are given by ${ }^{4}$

$$
S_{r}=\frac{\dot{\omega}_{c}}{\rho|\nabla c|}, S_{n}=\frac{\mathbf{N} \cdot \nabla(\rho D \mathbf{N} \cdot \nabla c)}{\rho|\nabla c|}, S_{t}=-2 D \kappa_{m},
$$

where $\kappa_{m}=0.5 \nabla \cdot \mathbf{N}$ is the flame curvature and $\mathbf{N}=-\nabla c /|\nabla c|$ is the flame normal vector.

The evolution equation of $|\nabla c|$ is given as ${ }^{12,13,21}$

$$
\frac{\partial|\nabla c|}{\partial t}+\frac{\partial\left(u_{j}|\nabla c|\right)}{\partial x_{j}}=a_{T}|\nabla c|-\frac{\partial\left(S_{d} N_{j}|\nabla c|\right)}{\partial x_{j}}+2 S_{d} \kappa_{m}|\nabla c|,
$$

where $a_{T}=\left(\delta_{i j}-N_{i} N_{j}\right)\left(\partial u_{i} / \partial x_{j}\right)$ is the tangential strain rate. Equation (5) can be rearranged as

$$
\frac{\partial|\nabla c|}{\partial t}+V_{j}^{c} \frac{\partial|\nabla c|}{\partial x_{j}}=-a_{N}|\nabla c|-N_{j} \frac{\partial S_{d}}{\partial x_{j}}|\nabla c|
$$

or

$$
\frac{1}{|\nabla c|} \frac{d|\nabla c|}{d t}=-a_{N}+N_{j} \frac{\partial S_{d}}{\partial x_{j}}=-a_{N}^{e f f},
$$

where $V_{j}^{c}=\left(u_{j}+S_{d} N_{j}\right)$ is the $j$-th component of propagation velocity of a given $c$ isosurface, $a_{N}=N_{i} N_{j} \partial u_{i} / \partial x_{j}$ is the flame normal strain rate, $a_{N}^{\text {eff }}$ is the effective normal strain rate that influences the evolution of $|\nabla c|$, and $d / d t=\partial() / \partial t+V_{j}^{c} \partial(\ldots)$ is the total derivative associated with flame movement. It was demonstrated by Dopazo et al. ${ }^{18}$ that $a_{N}^{\text {eff }}$ can alternatively be expressed as $a_{N}^{\text {eff }}$ $=\left(\Delta x_{N}\right)^{-1}\left(d \Delta x_{N} / d t\right)$, where $\Delta x_{N}$ is the normal distance between two $c$ isosurfaces. This suggests that a positive value of $a_{N}^{\text {eff }}$ acts to increase $\Delta x_{N}$, which, in turn, leads to a drop in $|\nabla c|$. Similarly, a negative value of $a_{N}^{\text {eff }}$ acts to decrease $\Delta x_{N}$ and promotes an increase in $|\nabla c|$. In this regard, it is also useful to consider the evolution of the flame surface area, $A,{ }^{10,11,18,46}$

$$
\frac{1}{A} \frac{d A}{d t}=a_{T}+2 S_{d} \kappa_{m}=\left(\delta_{i j}-N_{i} N_{j}\right) \frac{\partial u_{i}}{\partial x_{j}}+2 S_{d} \kappa_{m}=a_{T}^{e f f} .
$$

In Eq. (7), $2 S_{d} \kappa_{m}$ is the tangential strain rate due to flame propagation, and $a_{T}^{\text {eff }}$ is the effective tangential strain rate. ${ }^{18,46}$ The quantities $a_{T}^{e f f}$ and $2 S_{d} \kappa_{m}$ are alternatively referred to as stretch rate and curvature stretch, respectively. ${ }^{10,11}$ Note that in the following analysis, the statistics of the SDF have been investigated for RPVs based on $\mathrm{H}_{2}, \mathrm{O}_{2}$, and $\mathrm{H}_{2} \mathrm{O}$. In the present work, only the mean values of the terms on the right-hand sides of Eqs. (6) and (7) have been analyzed because the current analysis focuses on the statistical behaviors of the SDF and different strain rates, which affect the SDF evolution. This is consistent with several previous analyses, ${ }^{15,18,24,46}$ where a similar approach has been adopted.

\section{RESULTS AND DISCUSSION}

\section{A. Flow behavior}

Figure 2 shows the instantaneous isosurface of the temperature at $1700 \mathrm{~K}$ and the vorticity magnitude field within the flashback region of the flow. It can be seen from Fig. 2 that the flame alters the boundary layer structure and the turbulence decays across the flame in the near wall region, whereas turbulence is generated in the middle of the channel due to the merging of the two flame branches from the top and bottom walls. ${ }^{26}$ Figure 2 also shows the localized reverse flow regions of the flow (blue isosurfaces), which are clearly visible immediately upstream of each flame bulge and are limited to the near wall region. This behavior is consistent with the earlier findings of Gruber et al. ${ }^{4}$ The physical mechanism that leads to these reverse flow regions upstream of the flame has been discussed elsewhere $^{4,26}$ and thus is not repeated here. Figure 3 shows the progress variable computed from the $\mathrm{H}_{2}, \mathrm{O}_{2}$, and $\mathrm{H}_{2} \mathrm{O}$ mass fraction in the $x-y$ mid-plane of the channel. The differences in the location of the RPVs calculated from different species mass fractions are clearly visible at the trailing edge of the flame, which is typical of hydrogen flames. It can also be seen that the flame is highly stretched toward the middle of the channel due to the high flow velocity in the center $(y / h=1)$ of the channel. This leads to a slower propagation of the flame into the reactants at $y / h=1$, whereas the flame propagates at a much faster rate in the vicinity of the walls (at $y / h=0$ and $y / h=2)$. This raises the possibility of the differences in the behavior of the displacement speed at different $y / h$ locations in the channel. In this work, the results are reported at $y / h=0.005,0.1,0.5$, and 1 ; which in terms of the non-dimensional wall distance in the nonreacting channel $\left[y^{+}=\left(\rho u_{\tau} y\right) / \mu\right]$ is representative of $y^{+}=0.6,12,60$, and 120 , respectively. surfaces of the temperature at $1700 \mathrm{~K}$ (colored in red) and the instantaneous normalized vorticity magnitude in the flashback region of the channel. The negative flow velocity regions are shown on the top and bottom walls (blue color). 


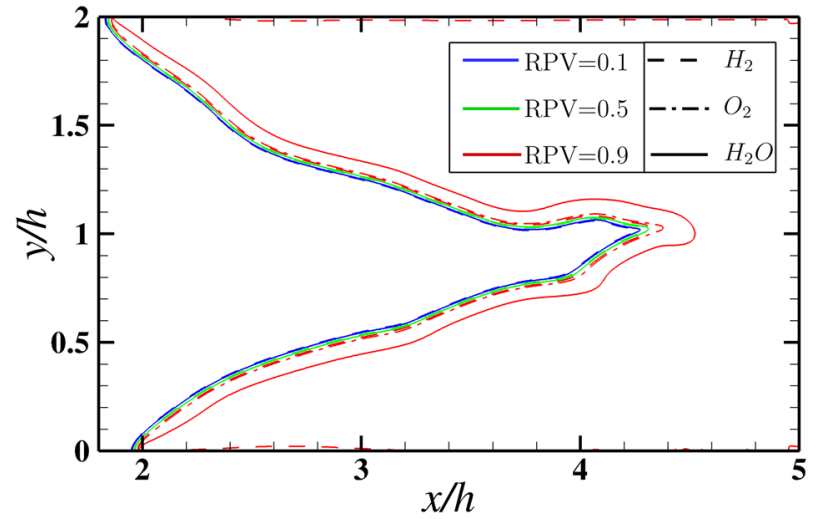

FIG. 3. The instantaneous reaction progress variable (RPV) based on the $\mathrm{H}_{2}, \mathrm{O}_{2}$, and $\mathrm{H}_{2} \mathrm{O}$ mass fraction in the $x-y$ mid-plane of the flashback region of the channel.

\section{B. Mean behavior of SDF and flame thickness}

The thickness of the reaction layer in a mean sense under turbulent conditions is important for the understanding of the flame surface area. In this context, the flame thickness is commonly determined by the mean gradient of $c$. Figure 4 shows the values of $|\nabla c|$ $\times \delta_{\text {th }}$ conditioned on $c$ at different wall distances for the RPV based on $\mathrm{H}_{2}, \mathrm{O}_{2}$, and $\mathrm{H}_{2} \mathrm{O}$. The maximum value of the profile for the mean value of $|\nabla c| \times \delta_{t h}$ conditioned upon $c$ is henceforth referred to as the peak mean value of the SDF in this work. Figure 4 shows that the value of $c$ at which the peak mean value of the SDF is obtained can be different for different choices of the RPV. Note that the peak mean value of $|\nabla c| \times \delta_{t h}$ indicates a propensity toward flame thinning or thickening in a mean sense relative to the unstrained freely propagating laminar flame conditions. ${ }^{21-24}$ In addition to the averaging method, as illustrated by $\mathrm{Yu}$ et al., ${ }^{47}$ the flame thickening vs thinning depends on the choice of the species used for $c$, as shown in Fig. 4. The peak mean value of $|\nabla c| \times \delta_{t h}$ increases (thinner flame) up to $y / h=0.5$ for all species and then decreases toward the middle of the channel. Furthermore, $\mathrm{H}_{2}$ and $\mathrm{O}_{2}$ based RPVs show a thinner flame when compared with the $\mathrm{H}_{2} \mathrm{O}$ based RPV across all the $y / h$ locations considered here. The variation in the SDF for all the RPVs at different $y / h$ arises due to the changes in the levels of turbulence experienced by the flame in the turbulent boundary layer. The flow is laminar in the vicinity of the wall, and a higher level of turbulence exists at $y / h=0.5$ before decreasing again toward the middle of the channel. ${ }^{26}$ Furthermore, the wall temperature plays an important role in determining the SDF based on different species in the near wall region.

\section{Mean behavior of aerodynamic strain and dilatation rates}

The variation in the SDF (and also the reaction zone thickness) with different species can be explained via Eqs. (5)-(7), which provide a means to analyze the specific contributions from the statistical behaviors of $S_{d}, a_{N}$, and $a_{T}$ to the SDF and its evolution. The instantaneous $c=0.5$ isosurfaces for different definitions of the RPV in the bottom half of the channel colored by $a_{N}, a_{T}$, and $\nabla \cdot \mathbf{u}$ are shown in Fig. 5. The dilatation rate $\nabla \cdot \mathbf{u}=a_{T}+a_{N}$ is vanishingly small
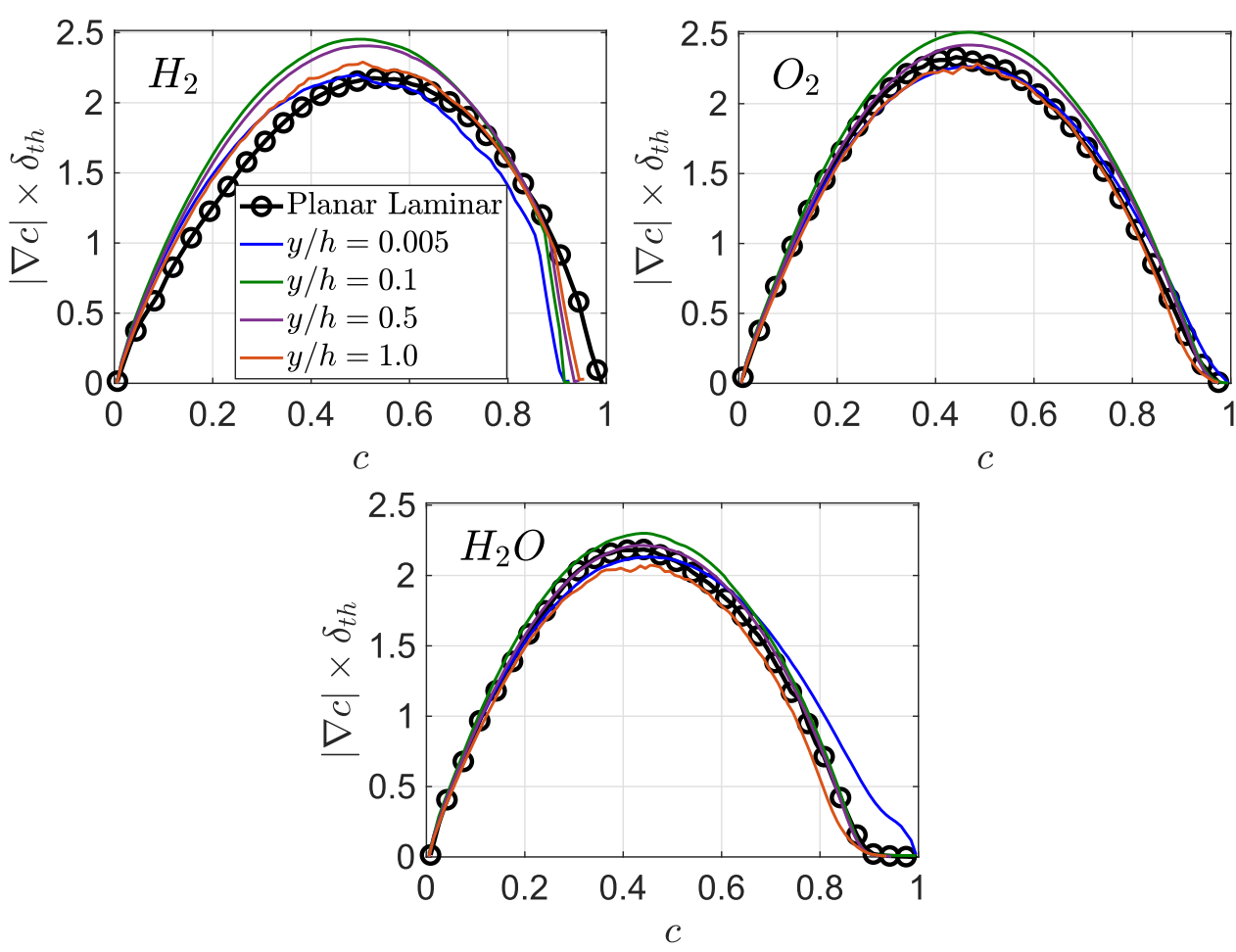

FIG. 4. Variations of the mean values of $|\nabla c| \times \delta_{\text {th }}$ conditioned on $c$ for all the RPVs considered at different $y / h$ locations. 

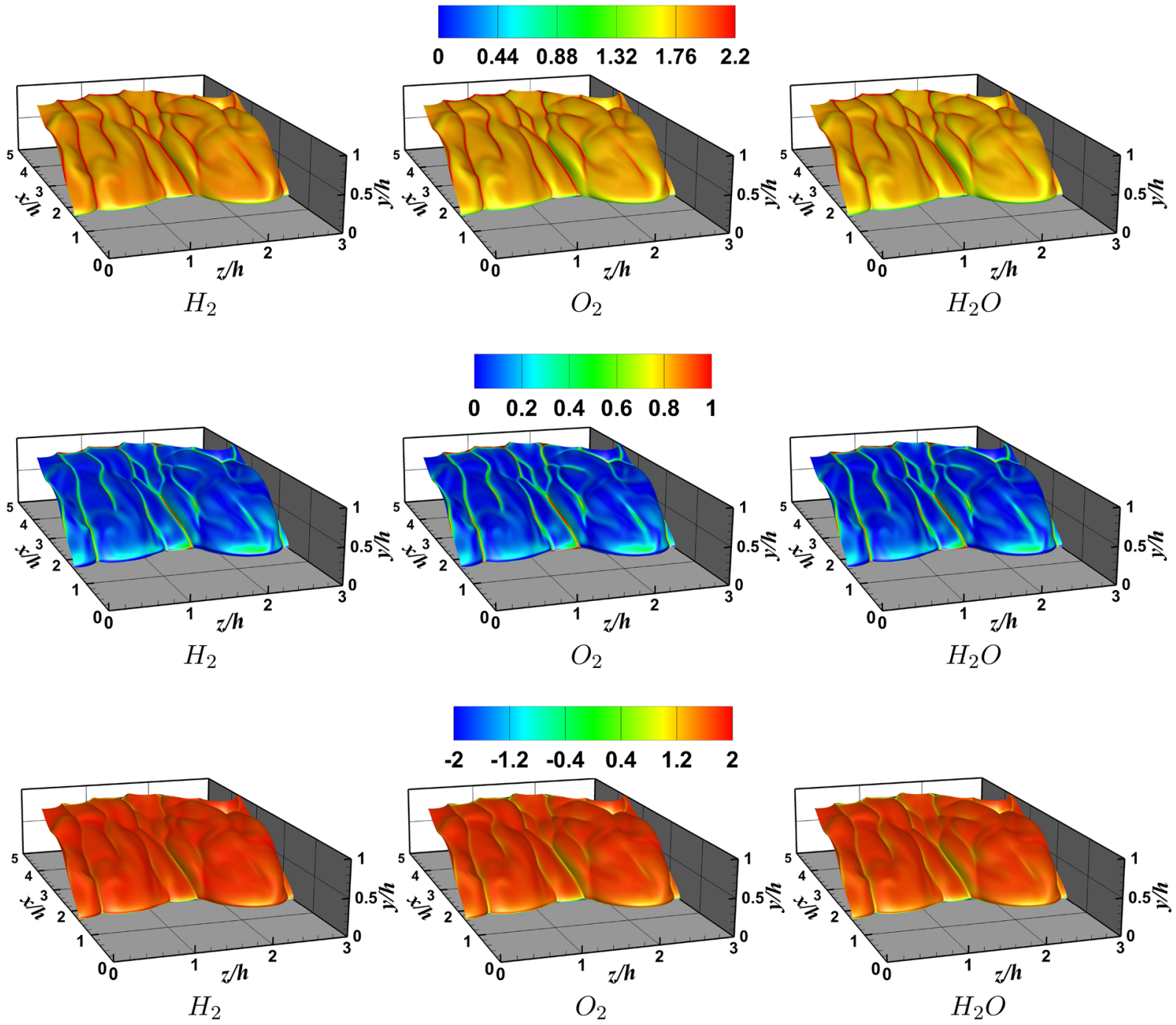

FIG. 5. Bottom half of the instantaneous turbulent flame represented by the $c=0.5$ isosurface colored by $\nabla \cdot \mathbf{u} \times \delta_{t h} / S_{L}$ (top row), $a_{T} \times \delta_{t h} / S_{L}$ (middle row), and $a_{N} \times \delta_{t h} / S_{L}$ (bottom row) for $\mathrm{H}_{2}, \mathrm{O}_{2}$, and $\mathrm{H}_{2} \mathrm{O}$ based RPVs.

close to the wall and assumes high values away from the wall for all the RPVs considered in this work. The value of $a_{N}$ becomes negative close to the wall and assumes positive values toward the center of the channel, whereas $a_{T}$ is maximum in the near wall region and assumes vanishingly small values away from the wall. The low level of variation in these statistics in the wall normal direction exists due to the low $R e_{\tau}$ of the non-reacting channel flow. Figure 6 shows that the mean values of dilatation rate $\nabla \cdot \mathbf{u}$, normal strain rate $a_{N}$, and the tangential strain rate $a_{T}$ conditioned upon $c$ for different choices of the RPV. In the near wall region, the mean values of $\nabla \cdot \mathbf{u}$ remain lower when compared with $a_{T}$ and $a_{N}$. This trend changes as the distances from the wall increases and $\nabla \cdot \mathbf{u}$ takes higher values, while the contribution from $a_{T}$ decreases. The dilatation rate $\nabla \cdot \mathbf{u}$ assumes mostly positive values in premixed flames due to heat release, but the effect is attenuated in the near wall region due to the cold wall and also due to the constriction of the velocity gradient in the wall normal direction.

The mean value of $a_{N}$ remains positive away from the wall, but in the near wall region, it assumes negative values. The normal strain rate $a_{N}$ can be expressed as $a_{N}=\left(e_{\alpha} \cos ^{2} \alpha+e_{\beta} \cos ^{2} \beta\right.$ $+e_{\gamma} \cos ^{2} \gamma$ ), where $e_{\alpha}, e_{\beta}$, and $e_{\gamma}$ are the most extensive (i.e., most $+v e$ ), intermediate, and most compressive (i.e., most -ve) principal strain rates, respectively, and $\alpha, \beta$, and $\gamma$ are the angles between $\nabla c$ and the eigenvectors associated with $e_{\alpha}, e_{\beta}$, and $e_{\gamma}$, respectively. It is well known that $\nabla c$ aligns with the $e_{\alpha}$-eigenvector when the strain rate due to heat release dominates over turbulent straining. ${ }^{13,16,17}$ In contrast, $\nabla c$ preferentially aligns with the $e_{\gamma}$-eigenvector when the turbulent strain rate overcomes the strain rate arising from heat release. $^{13,16,17}$ The trends for the alignment between the strain rate eigenvectors and the gradient of the progress variable are presented 

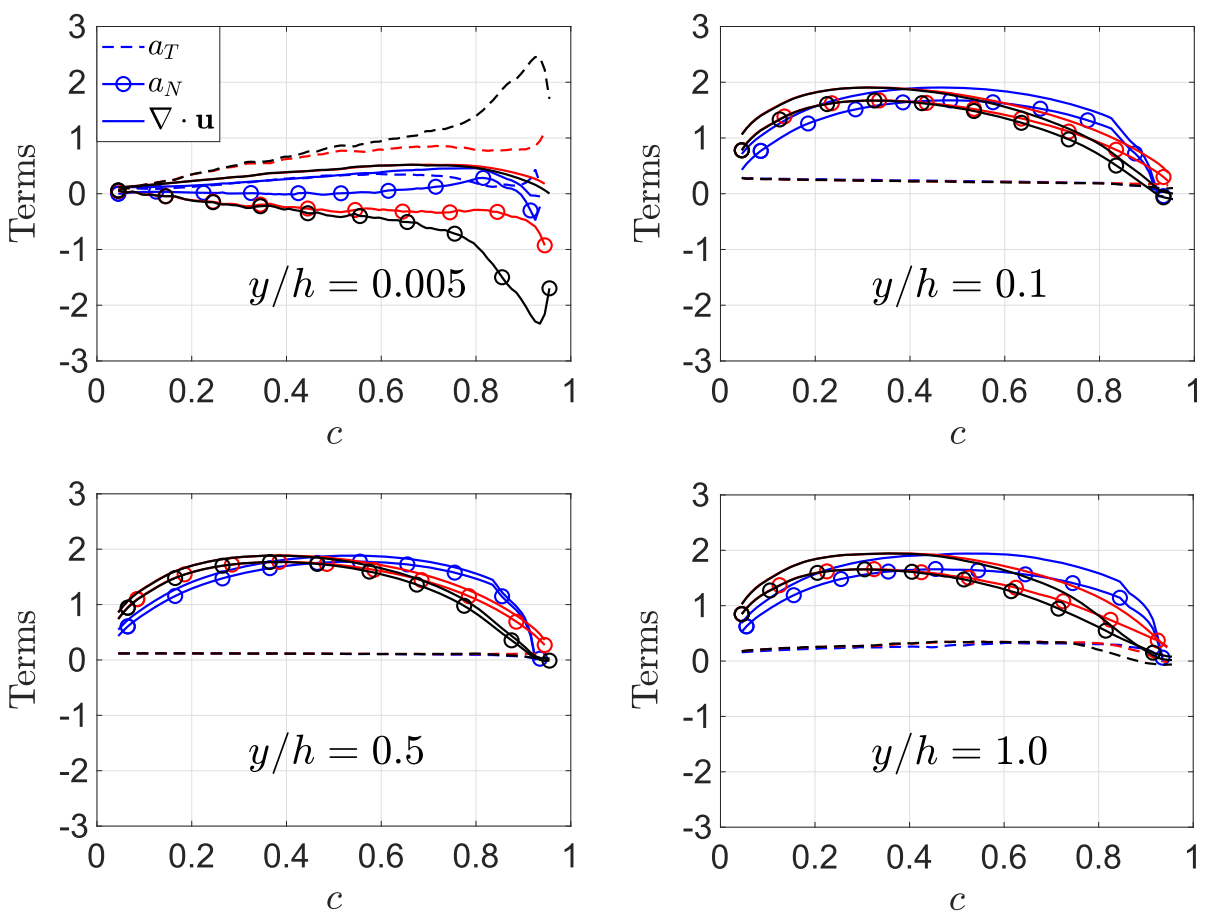

FIG. 6. Profiles of the mean values of $\nabla \cdot \mathbf{u}, a_{N}$, and $a_{T}$ normalized by $\delta_{t h} / S_{L}$ conditioned on $c$ at different $y / h$ locations. The different colors represent RPV definitions based on $\mathrm{H}_{2}$ (blue lines), $\mathrm{O}_{2}$ (red lines), and $\mathrm{H}_{2} \mathrm{O}$ (black lines).

in Fig. 7 , where the values for $e_{\alpha} \cos ^{2} \theta_{\alpha}, e_{\beta} \cos ^{2} \theta_{\beta}$, and $e_{\gamma} \cos ^{2} \theta_{\gamma}$ are shown at different $y / h$ locations. In the current analysis, the strain rate arising due to heat release remains low in the near wall region because of the low temperature at the wall leading to a small reaction rate magnitude and a weakening of the strain rate arising from thermal expansion. This consequently leads to $\nabla c$ aligning with $e_{\gamma}$-eigenvector, thus giving rise to negative mean values of $a_{N}$. In the regions away from the wall, the strain rate arising due to
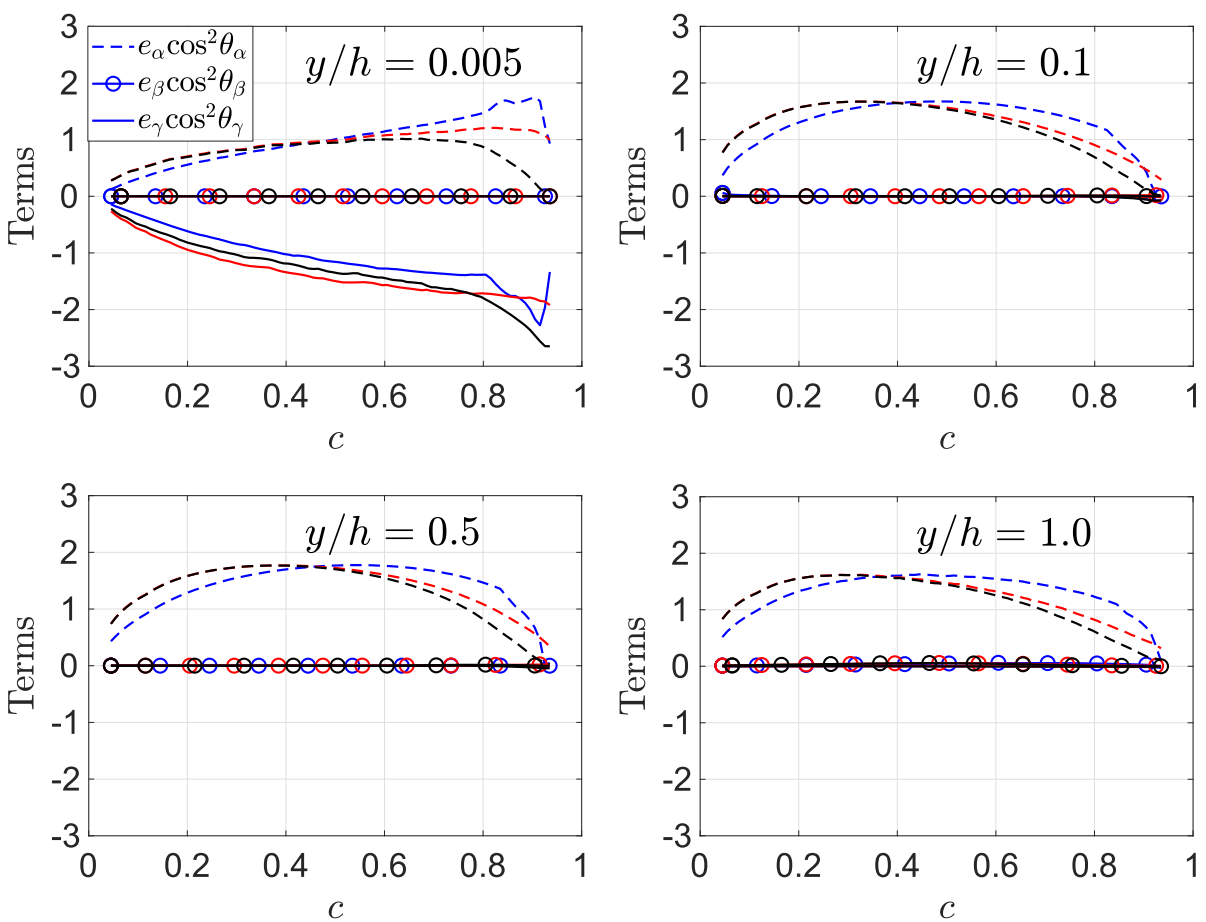

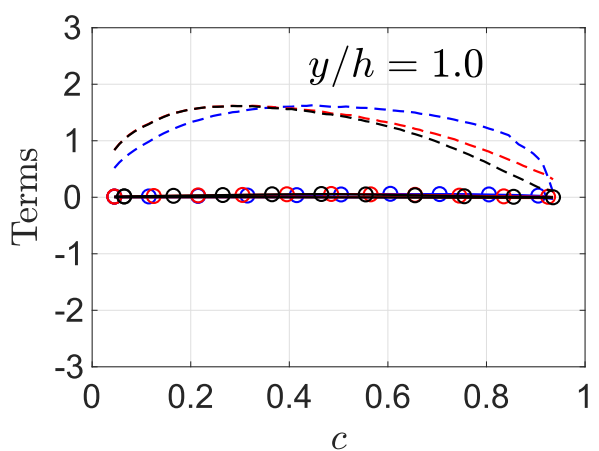

FIG. 7. Profiles of the mean values of $e_{\alpha} \cos ^{2} \theta_{\alpha}, e_{\beta} \cos ^{2} \theta_{\beta}$, and $e_{\gamma} \cos ^{2} \theta_{\gamma}$ normalized by $\delta_{t h} / S_{L}$ conditioned on $c$ at different $y / h$ locations. The different colors represent RPV definitions based on $\mathrm{H}_{2}$ (blue lines), $\mathrm{O}_{2}$ (red lines), and $\mathrm{H}_{2} \mathrm{O}$ (black lines). 
heat release remains high, and $\nabla c$ aligns with the $e_{\alpha}$-eigenvector, as shown in Fig. 7, and leads to positive mean values of $a_{N}$. From Eq. (5), it can be inferred that in the regions away from the wall, the normal flow strain has a net flame thickening effect, whereas in the near wall region, it has a flame thinning effect. It should be noted here that this qualitative behavior is independent of the definition of the RPV.

The influence on the flame surface can be determined by examining the behavior of $a_{T}$. The mean value of $a_{T}=\nabla \cdot \mathbf{u}-a_{N}$ is determined by the relative magnitudes and signs of $\nabla \cdot \mathbf{u}$ and $a_{N}$. In the near wall region, the large negative mean value of $a_{N}$ and a small mean positive value of $\nabla \cdot \mathbf{u}$ lead to a large positive mean value of $a_{T}=\nabla \cdot \mathbf{u}-a_{N}$. According to Eq. (7), this implies that $a_{T}$ strengthens in the near wall region, leading to a higher flame surface area. In the regions away from the wall, the mean values of $\nabla \cdot \mathbf{u}$ and $a_{N}$ remain close to each other, leading to a small positive mean value of $a_{T}$.

\section{Mean behavior of displacement speed}

Figure 8 shows the mean values of $S_{d} / S_{L}$ conditioned on $c$, which are representative of the effects associated with flame propagation. The mean value of $S_{d} / S_{L}$ increases from the unburned to the burned gas side of the flame-front for all the species except for $\mathrm{H}_{2}$, where the mean value of $S_{d} / S_{L}$ decreases sharply at high values of $c$ in the near wall region due to the heat loss at the wall. Further away from the wall, the mean $S_{d}$ increases toward the middle of the channel, and the peak mean value of $S_{d} / S_{L}$ is observed close to the middle of the flame-front but slightly skewed toward the products.
The differences in the mean behaviors of $S_{d} / S_{L}$ for different species can be explained by examining the statistical behaviors of $S_{r}$, $S_{n}$, and $S_{t}$. The variations of $S_{r} / S_{L}, S_{n} / S_{L}$, and $S_{t} / S_{L}$ conditioned on $c$ are shown in Fig. 9. The mean values of $\left(S_{r}+S_{n}\right) / S_{L}$ (not shown here) and $S_{d} / S_{L}$ remain close to each other as the mean contribution of $S_{t}=-2 D \kappa_{m}$ remains negligibly small in this case. Figure 9 shows that the mean value of $S_{r} / S_{L}$ remains positive throughout the flame-front, whereas the mean value of $S_{n} / S_{L}$ assumes positive (negative) values toward the unburned (burned) gas side of the flame-front. Note that the qualitative distributions of $S_{r} / S_{L}$ and $S_{n} / S_{L}$ are different for different species at all distances away from the wall, which contribute to the differences in $S_{d} / S_{L}$ for different RPV definitions and are consistent with the earlier findings from the statistically planar turbulent premixed flames. ${ }^{1}$

\section{E. Mean behavior of the strain rates due to flame propagation}

In order to assess the contributions from the flame propagation to the SDF, as appearing in Eq. (5), the mean values of $N_{j} \partial S_{d} / \partial x_{j}$ normalized with $\delta_{t h} / S_{L}$ conditioned on $c$ for different choices of the RPV at different $y / h$ locations are shown in Fig. 10. The mean value of $N_{j} \partial S_{d} / \partial x_{j}$ is dominated by $N_{j} \partial\left(S_{r}+S_{n}\right) / \partial x_{j}$, while the effect of $N_{j} \partial S_{t} / \partial x_{j}$ is negligible for all RPVs at all locations, as shown in Fig. 11. In the near wall region, $N_{j} \partial S_{d} / \partial x_{j}$ remains close to zero for all RPVs. At $y / h=0.1, N_{j} \partial S_{d} / \partial x_{j}$ becomes negative for all RPVs, which acts to promote flame thinning. Toward the middle of the channel, $N_{j} \partial S_{d} / \partial x_{j}$ shows both positive and negative values for all RPVs, which implies local thickening and thinning of the flame due to the merging of the two flame branches from the top and
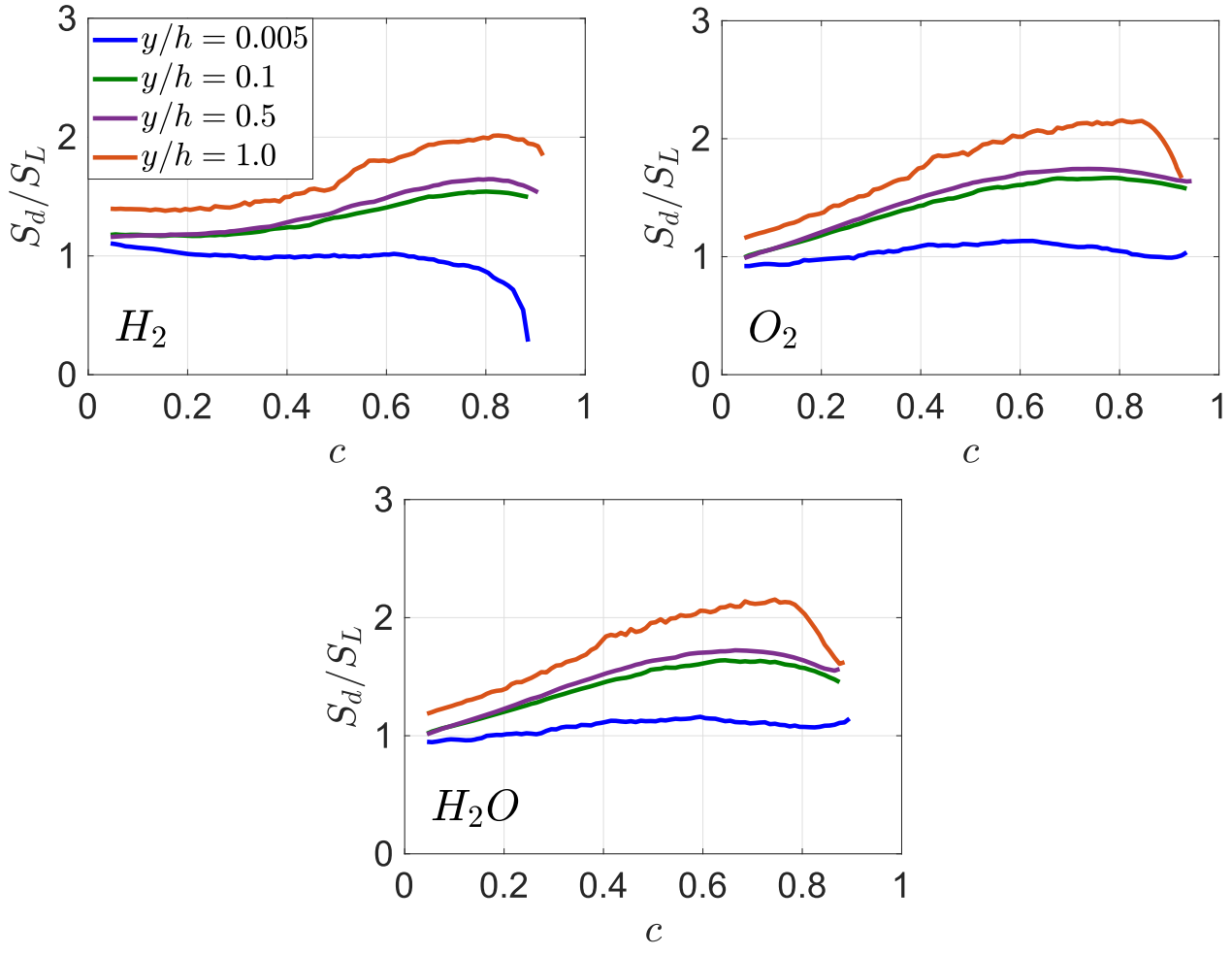

FIG. 8. Profiles of the mean values of $S_{d} / S_{L}$ conditioned on $c$ for different RPVs at different $y / h$ locations. 

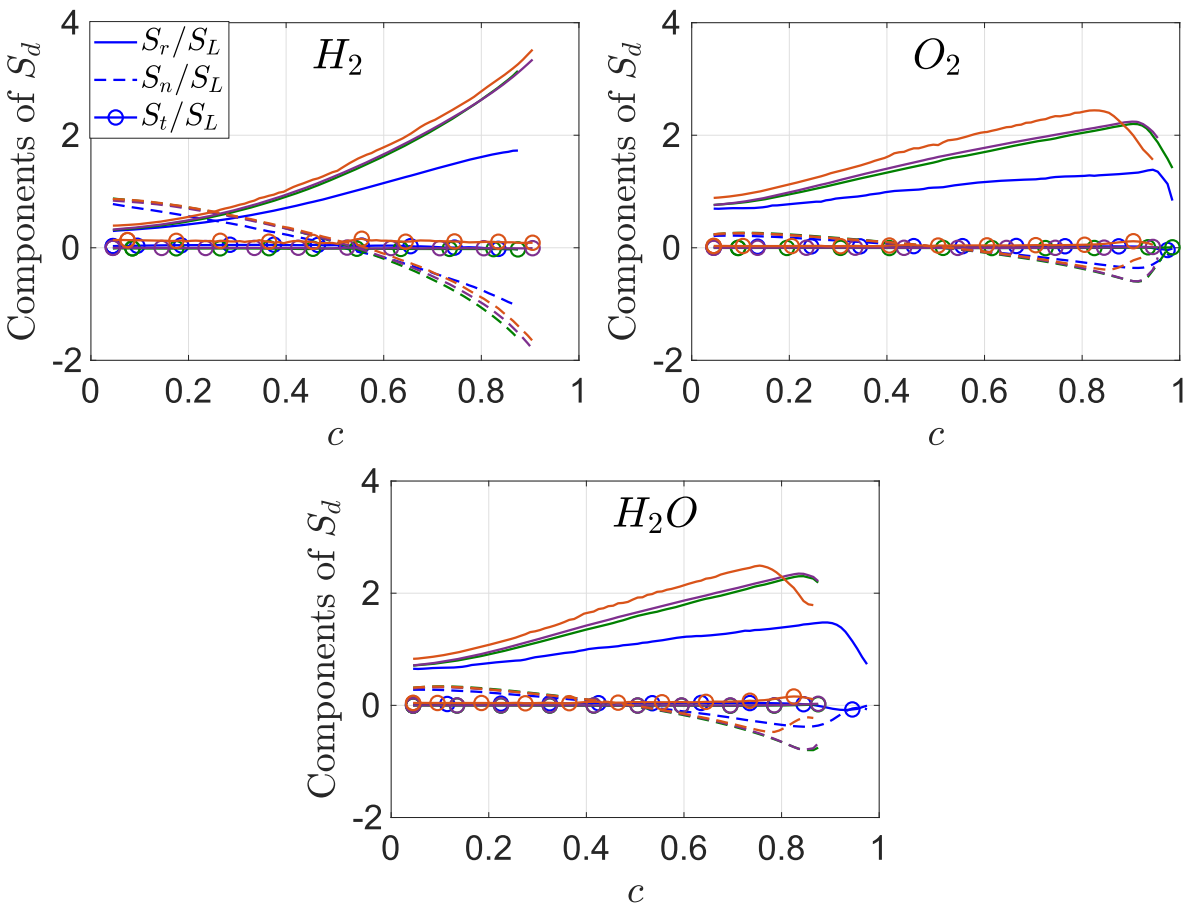

FIG. 9. Profiles of the mean values of the components of $S_{d} / S_{L}$ conditioned on $c$ at different $y / h$ locations. The blue, green, purple, and orange colors denote $y / h=0.005,0.1,0.5$, and 1.0 locations, respectively. bottom walls, as shown in Fig. 3. The behavior of the flame propagation effects associated with the curvature stretch $2 S_{d} \kappa_{m}$, last term in Eq. (5), is also shown in Fig. 10. Note that $2 S_{d} \kappa_{m}$ and its components, as shown in Fig. 12, remain predominantly negative at all $y / h$ locations investigated in this work. This is due to the contribution arising from the tangential diffusion component of displacement speed (i.e. $2 S_{t} \kappa_{m}=-4 D \kappa_{m}^{2}$ ) and also owing to the negative or very small positive values of $\kappa_{m}$ at all $y / h$ locations investigated. The
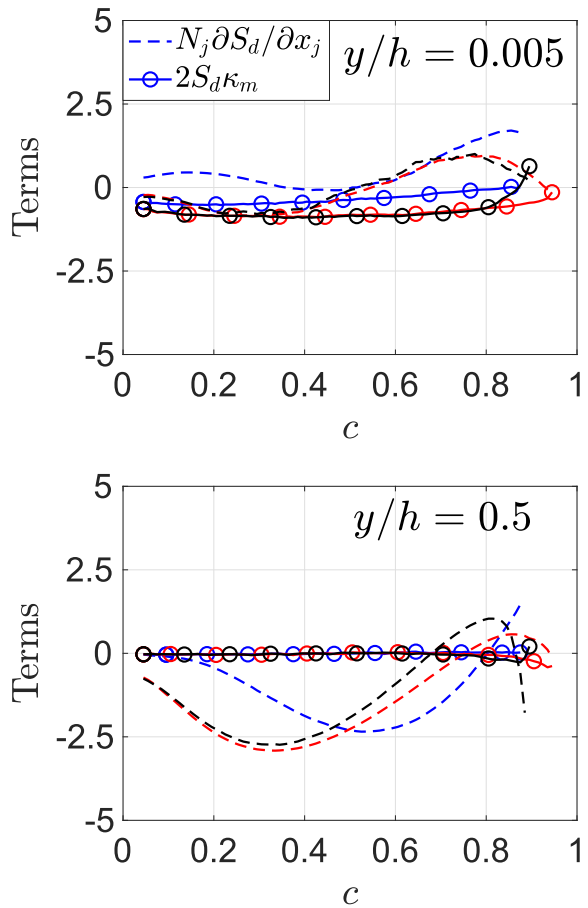
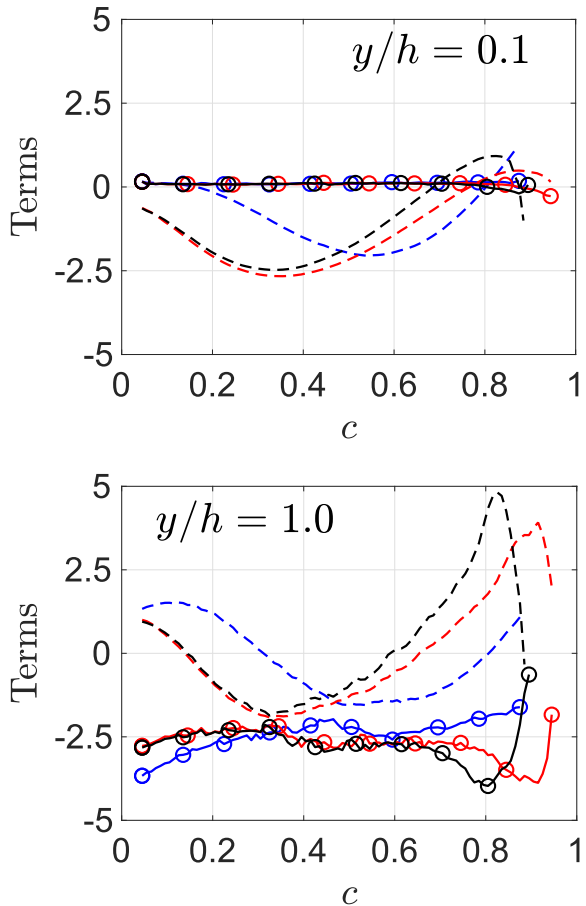

FIG. 10. Profiles of the mean values of $N_{j} \partial S_{d} / \partial x_{j}$ and $2 S_{d} \kappa_{m}$ normalized by $\delta_{t h} / S_{L}$ at different $y / h$ locations. The blue, red, and black colors denote RPVs based on $\mathrm{H}_{2}, \mathrm{O}_{2}$, and $\mathrm{H}_{2} \mathrm{O}$, respectively. 

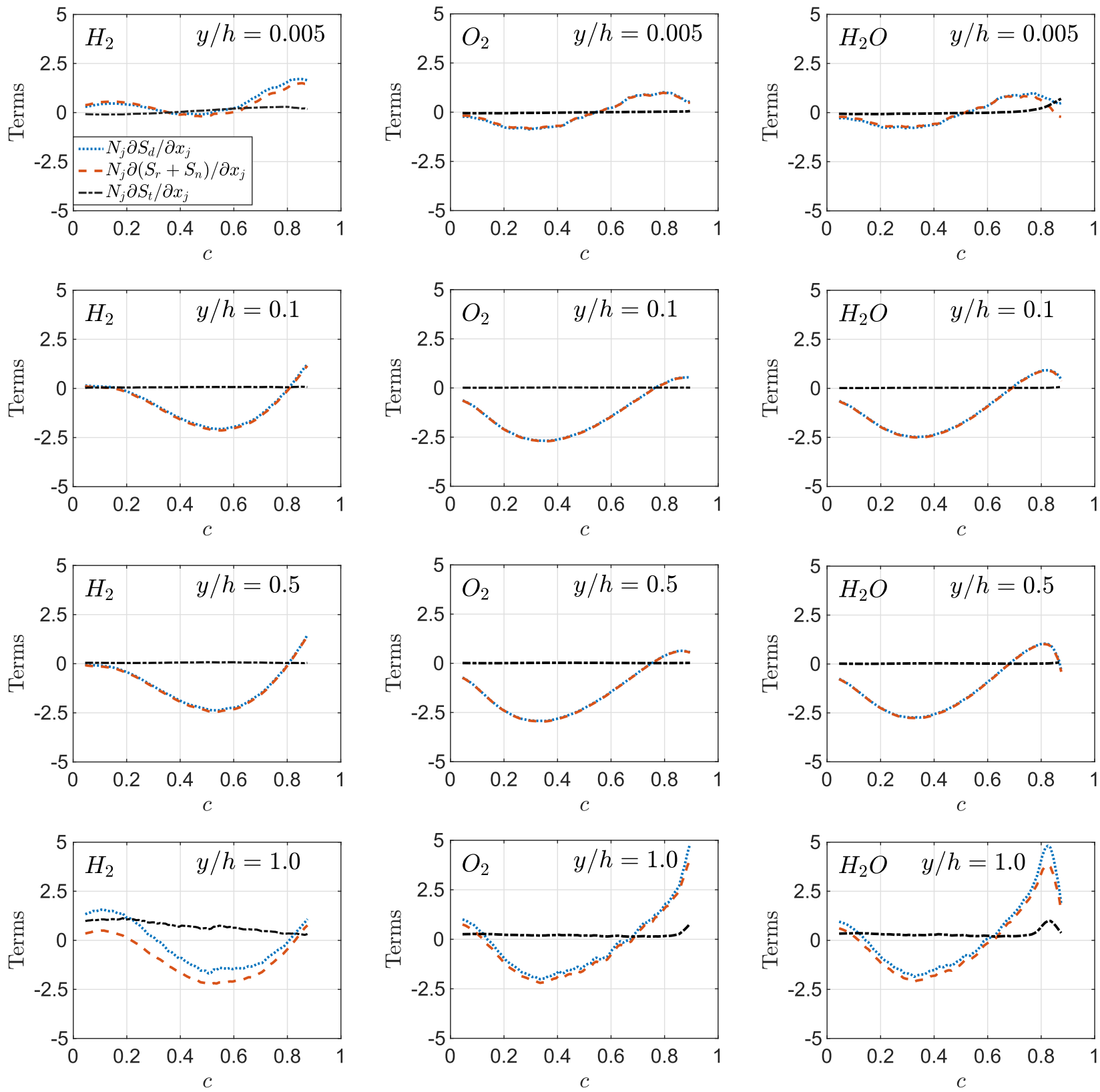

FIG. 11. Profiles of the mean values of $N_{j} \partial S_{\alpha} / \partial x_{j}(\alpha=d,(r+n)$ and $t)$ normalized by $\delta_{t h} / S_{L}$ conditioned on $c$ at different $y / h$ locations for $\mathrm{H}_{2}, \mathrm{O}_{2}$, and $\mathrm{H}_{2} \mathrm{O}$ based RPVs.

differences in $S_{d}$ statistics and $c$ distributions contribute to the qualitative and quantitative differences in the mean behaviors of $N_{j} \partial S_{d} / \partial x_{j}$ and $2 S_{d} \kappa_{m}$ for different RPV definitions.

\section{F. Mean behavior of effective normal and tangential strain rates}

The mean values of the effective normal strain rate $a_{N}^{\text {eff }}$ conditioned on $c$ for different RPV choices at different $y / h$ locations are presented in Fig. 13. According to Eq. (6), the effective normal strain rate assumes both positive and negative values within the flame-front at $y / h=0.005,0.1$, and 0.5 locations, whereas at $y / h=1.0 a_{N}^{e f f}$, the effective normal strain rate assumes only positive values for all RPVs. It is also important to appreciate that an averaging of $a_{N}^{e f f}$ and $a_{T}^{\text {eff }}$ does not yield information about mean values of $|\nabla c|$ and $A$ because the mean values of $|\nabla c|$ and $A$ cannot easily be extracted from the mean values of $|\nabla c|^{-1}(d|\nabla c| / d t)$ 

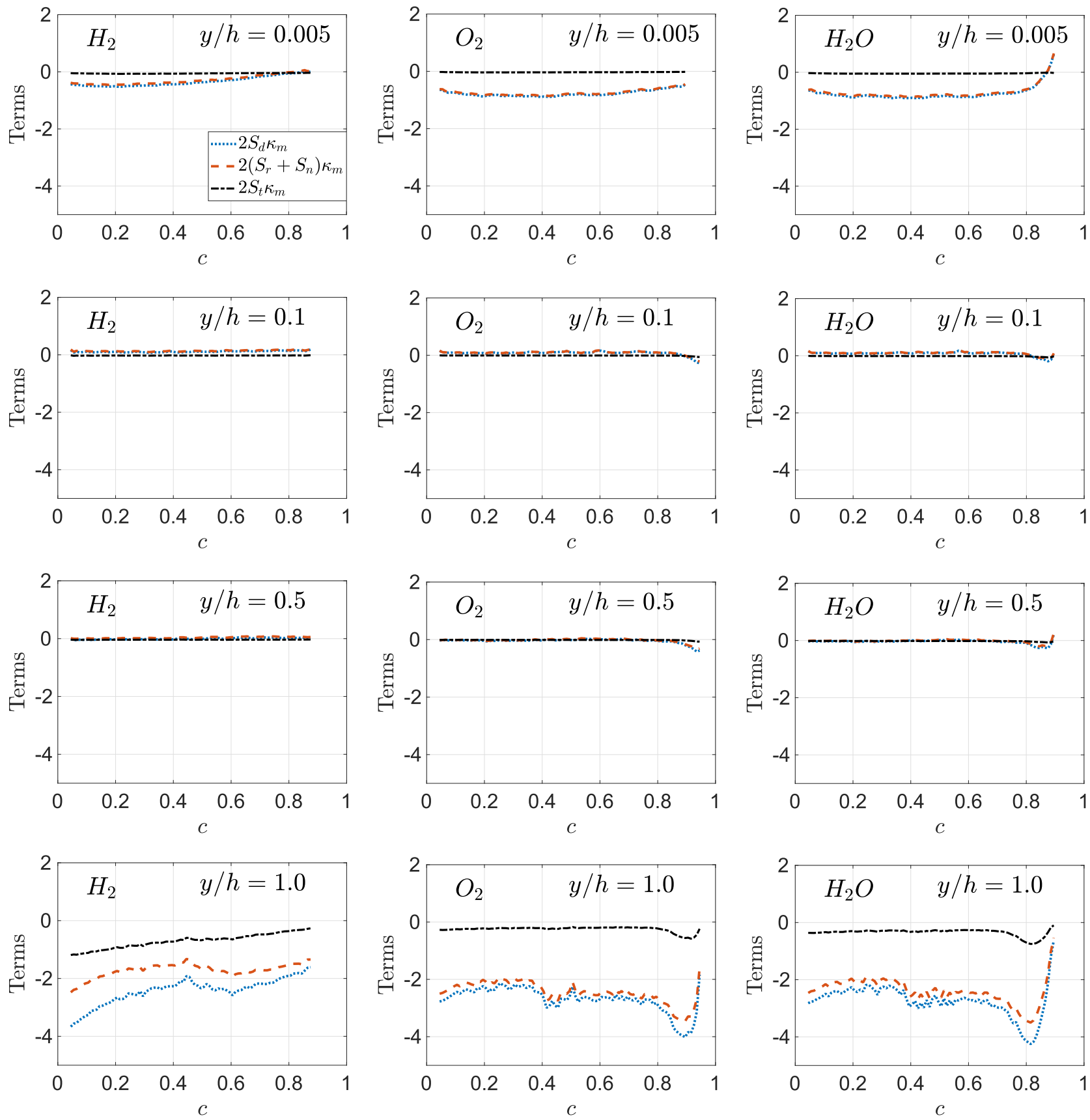

FIG. 12. Profiles of the mean values of $2 S_{\alpha} \kappa_{m}(\alpha=d,(r+n)$ and $t)$ normalized by $\delta_{t h} / S_{L}$ conditioned on $c$ at different $y / h$ locations for $\mathrm{H}_{2}, \mathrm{O}_{2}$, and $\mathrm{H}_{2} \mathrm{O}$ based RPVs.

and $A^{-1}(d A / d t)$ [see Eqs. (6) and (7)]. However, both $|\nabla c|$ and $A$ are positive quantities, and thus, positive values of $a_{N}^{\text {eff }}$ and $a_{T}^{\text {eff }}$ suggest a propensity of a decrease in $|\nabla c|$ and an increase in the flame surface area $A$, respectively. ${ }^{15,18,19,22-24,46}$ The changes in the SDF of the turbulent flame at a given $y / h$ location relative to the freely propagating planar laminar flame (i.e., $|\nabla c|_{T} /|\nabla c|_{L}$, where the subscripts $T$ and $L$ represent the values for turbulent and planar laminar flame, respectively) are shown in Fig. 14. A comparison between Figs. 13 and 14 reveals that the negative mean $a_{N}^{\text {eff }}$ occurs for the $y / h$ locations at which $|\nabla c|_{T} /|\nabla c|_{L}$ attains the highest value relative to the other $y / h$ locations, which is consistent with the propensity of obtaining higher values of $|\nabla c|$ in the turbulent flame 

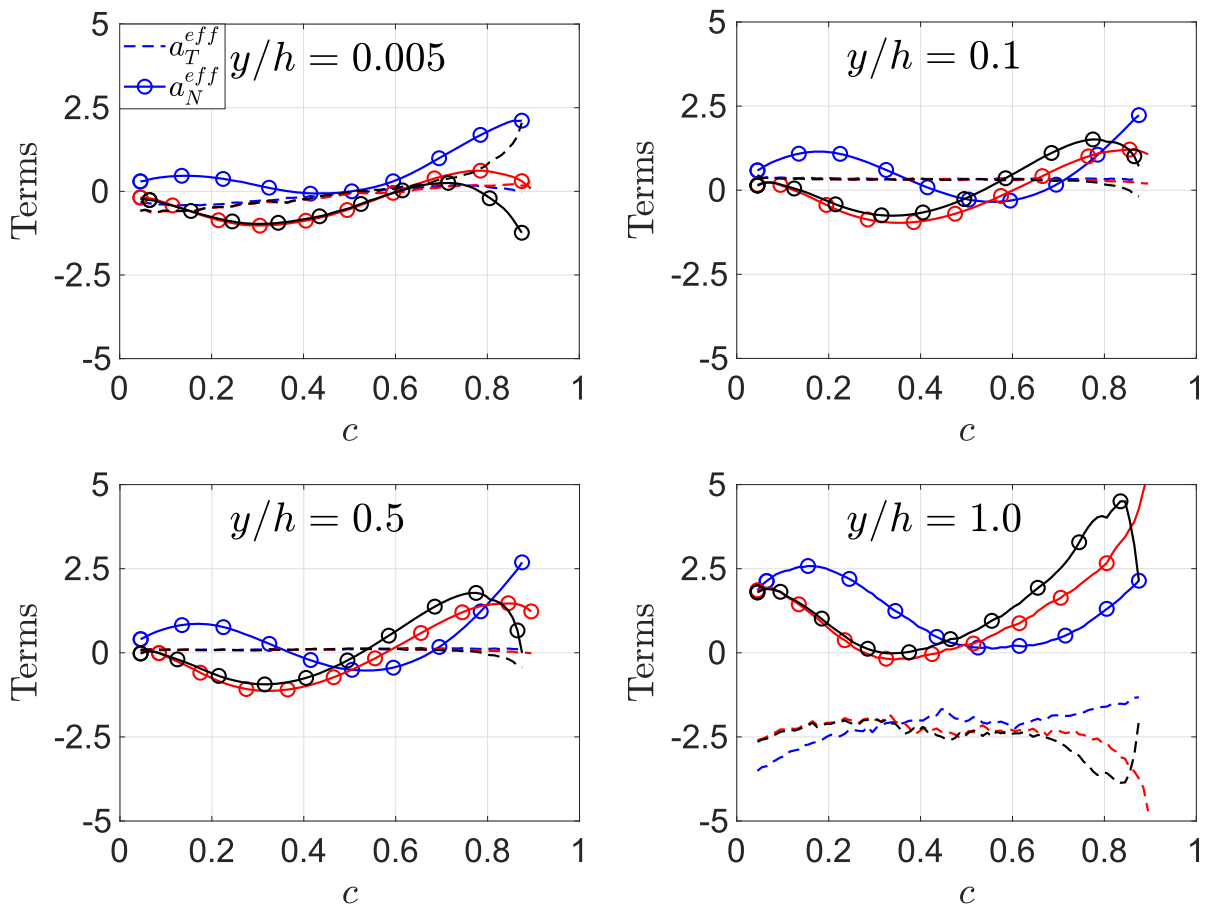

FIG. 13. Profiles of the mean values of $a_{N}^{\text {eff }}$ and $a_{T}^{\text {eff }}$ normalized by $\delta_{t h} / S_{L}$ at different $y / h$ locations. The blue, red, and black colors denote RPVs based on $\mathrm{H}_{2}$, $\mathrm{O}_{2}$, and $\mathrm{H}_{2} \mathrm{O}$, respectively.

than in the corresponding laminar flame, as observed in Fig. 4. By contrast, the positive mean $a_{N}^{e f f}$ occurs for the $y / h$ locations at which $|\nabla c|_{T} /|\nabla c|_{L}$ attains the lowest values relative to the other $y / h$ locations.
Figure 13 also shows the mean $a_{T}^{\text {eff }}$ conditioned on $c$ for different RPV choices at all $y / h$ locations. A positive (negative) value of $a_{T}^{\text {eff }}$ is indicative of flame area generation (destruction). Figure 13 shows that in the near wall region, the mean $a_{T}^{e f f}$ remains negative at small
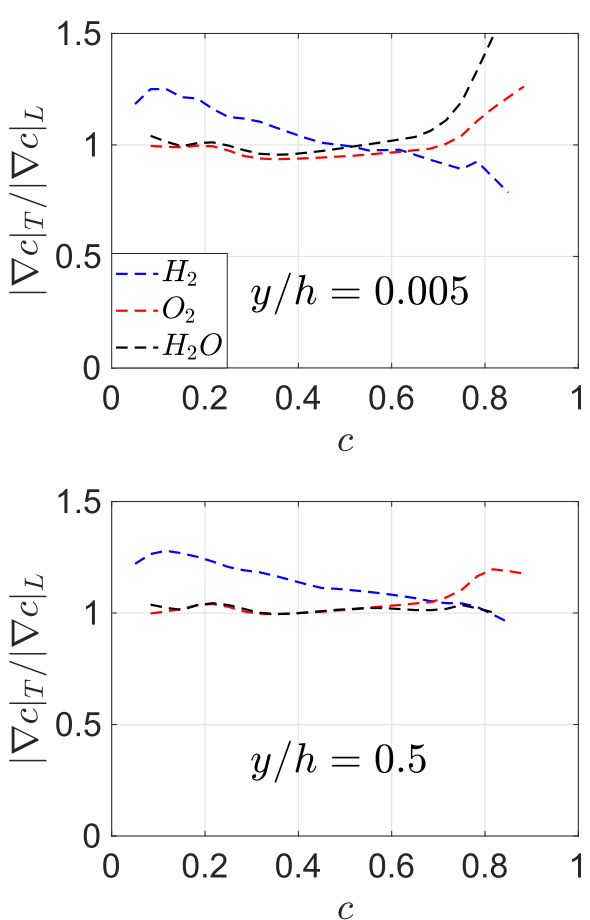
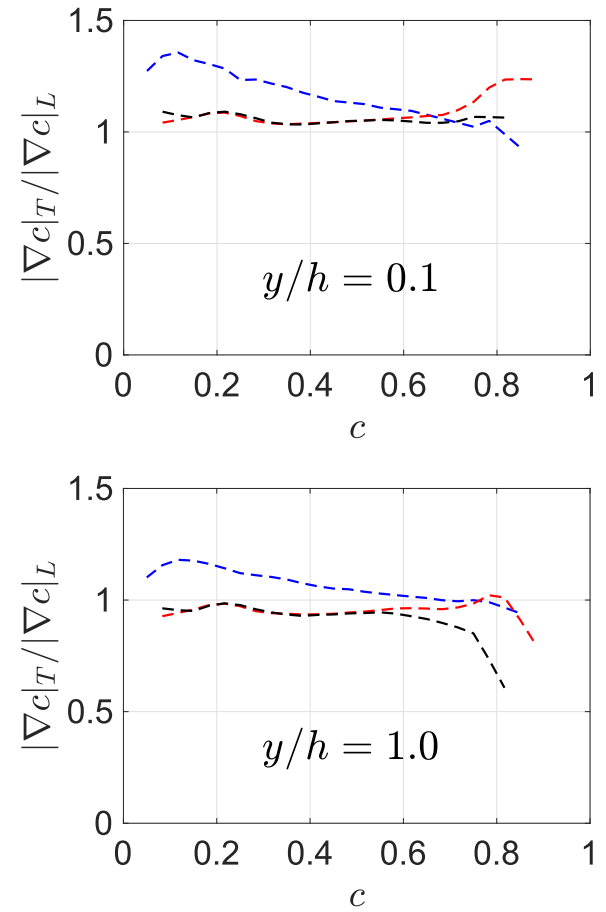

FIG. 14. Variations of the mean values of $|\nabla c|_{T} /|\nabla c|_{L}$, where subscripts $T$ and $L$ imply turbulent and planar laminar flame, conditioned on $c$ for all the RPVs considered at different $y / h$ locations. 
values of $c$ and becomes positive toward the higher values of $c$ for all RPVs considered, which implies that the flame surface is destroyed at the front of the flame due to the cold wall and the flame surface is generated in the reaction zone. Toward the middle of the channel, the mean $a_{T}^{\text {eff }}$ assumes small positive values within the flame-front, whereas at the middle of the channel, the mean $a_{T}^{\text {eff }}$ becomes negative for all the RPV definitions considered due to the merging of the top and bottom flame branches.

\section{G. Implications on modeling}

The statistical behaviors of the different strain rates, which affect the evolution of the SDF, as identified in subsections IV C-IV F, have a significant influence on the modeling of boundary layer flashback. The two most common approaches used for modeling in LES and RANS simulations of premixed turbulent flames are the generalized $\mathrm{FSD}^{7}$ (i.e., $\Sigma_{\text {gen }}=\overline{|\nabla c|}$ ) and the $\mathrm{SDR}^{8}$ (i.e., $N_{c}$ $\left.=D|\nabla c|^{2}\right)$ approaches. The transport equation of the generalized FSD can be obtained by multiplying Eq. (5) by $2|\nabla c|$ as ${ }^{48}$

$$
\begin{aligned}
\frac{\partial|\nabla c|^{2}}{\partial t} & =u_{j} \frac{\partial|\nabla c|^{2}}{\partial x_{j}} \\
& =-2 a_{N}|\nabla c|^{2}-2 N_{j} \frac{\partial S_{d}}{\partial x_{j}}|\nabla c|^{2}-S_{d} N_{j} \frac{\partial|\nabla c|^{2}}{\partial x_{j}},
\end{aligned}
$$

and upon Reynolds averaging/LES filtering, Eq. (8) leads to the transport equation for $\Sigma_{g e n}$. Furthermore, algebraic manipulation of Eq. (8) leads to the SDR transport equation as ${ }^{8,48}$

$$
\begin{aligned}
\rho \frac{\partial N_{c}}{\partial t}+\rho u_{j} \frac{\partial N_{c}}{\partial x_{j}}= & -2 \rho a_{N} N_{c}-2 \rho N_{j} \frac{\partial S_{d}}{\partial x_{j}} N_{c}-\rho S_{d} N_{j} \frac{\partial N_{c}}{\partial x_{j}} \\
& +\rho S_{d} N_{j} N_{c} \frac{1}{D} \frac{\partial D}{\partial x_{j}}+\frac{\rho N_{c}}{D}\left(\frac{\partial D}{\partial t}+u_{j} \frac{\partial D}{\partial x_{j}}\right)
\end{aligned}
$$

and further simplification of the expression in Eq. (9) leads to

$$
\begin{aligned}
\rho \frac{\partial N_{c}}{\partial t}+\rho u_{j} \frac{\partial N_{c}}{\partial x_{j}}= & -2 \rho a_{N}^{e f f} N_{c}-\rho S_{d} N_{j} \frac{\partial N_{c}}{\partial x_{j}} \\
& +\rho S_{d} N_{j} N_{c} \frac{1}{D} \frac{\partial D}{\partial x_{j}}+\frac{\rho N_{c}}{D}\left(\frac{\partial D}{\partial t}+u_{j} \frac{\partial D}{\partial x_{j}}\right) .
\end{aligned}
$$

It can be noticed in Eqs.(8)-(10) that the statistics of the strain rates $a_{N}, a_{T}, N_{j} \partial S_{d} / \partial x_{j}, 2 S_{d} \kappa_{m}, a_{N}^{e f f}$, and $a_{T}^{\text {eff }}$ play a significant role in the evolution of FSD and SDR. Furthermore, it should also be recognized that the choice of the RPV in the case of detailed chemistry simulations is important and should be accounted for in the modeling of SDR and FSD approaches, as suggested by the statistics presented in subsections IV C-IV F. The existence of the cold wall changes the displacement speed statistics in the near wall region, consequently altering the flame dynamics and the behavior of $|\nabla c|$. Moreover, the existence of the boundary layer and the heat loss at the wall changes the velocity gradient dynamics affecting the scalar gradient alignment with the strain rate eigenvectors, which consequently leads to changes in the normal and tangential strain rates. These effects need to be explicitly included in the FSD and SDR closure strategies for the accurate modeling of turbulent boundary layer flashback.

\section{CONCLUSIONS}

The mean behaviors of the SDF $(|\nabla c|)$ and the strain rates affecting $|\nabla c|$ transport have been analyzed for different RPV definitions by using a DNS database for flashback of the hydrogen-air premixed flame in a fully developed turbulent channel flow. The non-reacting turbulence characteristics of the channel flow are representative of the friction velocity based Reynolds number $R e_{\tau}=120$, while a hydrogen-air mixture with an equivalence ratio of 1.5 has been considered. A detailed chemical mechanism with nine species and twenty reactions is employed for an accurate representation of hydrogen-air combustion. The SDF and the strain rate statistics have been analyzed for RPVs based on $\mathrm{H}_{2}, \mathrm{O}_{2}$, and $\mathrm{H}_{2} \mathrm{O}$ mass fractions. The differences in $|\nabla c|$ statistics for different definitions of RPV have been explained in terms of the statistics of strain rates in the flame normal and tangential directions. It is found that the dilatation rate effects weaken in the near wall region due to the cold wall, and the alignment of $\nabla c$ with the most extensive (compressive) principal strain rate strengthens (weakens) as the distance from the wall increases. This leads to differences in the behavior of normal and tangential strain rates at different distances away from the wall. The mean behaviors of $S_{d}$ and its components for different choices of the RPV have been found to be qualitatively different. This leads to differences in the normal strain rate arising from flame propagation and the curvature stretch for different choices of the RPV. It is also found that the underlying turbulence in the boundary layer and the existence of the cold wall have a significant influence on the aforementioned statistics.

It should be recognized here that the results presented in this work may be sensitive to the $R e_{\tau}$ of the channel flow, and more data at different $R e_{\tau}$ need to be analyzed to investigate the influence of the variation in turbulence on SDF statistics. The findings show that the qualitative behaviors of the aerodynamic strain and dilatation rates do not depend on the choice of the RPV, but the mean behaviors of displacement speed and its components are affected by the choice of the RPV and have implications on the submodels used to close the mean/filtered reaction rate in RANS and LES approaches. These submodels usually rely on Flame Surface Density (FSD) ${ }^{7}$ or scalar dissipation rate $(\mathrm{SDR})^{8}$ based formulations. Hence, the models used to close the FSD and SDR transport equations need to accurately capture the respective behaviors of the unclosed terms for different definitions of the RPV at different distances away from the wall in the case of turbulent boundary layer flashback of premixed flames.

\section{ACKNOWLEDGMENTS}

This research was partially supported by the MEXT, Japan, as a "Priority issue on Post-K computer" (Accelerated Development of Innovative Clean Energy Systems) and used the computational resources of the K-computer provided by the RIKEN Advanced Institute for Computational Science through the HPCI System Research project (Project No. hp160220), ARCHER (Project Nos. EP/K025163/1 and EP/R029369/1), and the HPC facility at Newcastle University (Rocket). N.C. and U.A. are grateful to the EPSRC (Project No. EP/P022286/1) for financial support. U.A. acknowledges financial support from the JSPS (Fellowship ID No. PE18039). 


\section{DATA AVAILABILITY}

The data that support the findings of this study are available from the corresponding author upon reasonable request.

\section{REFERENCES}

${ }^{1} \mathrm{O}$. Bolland and $\mathrm{H}$. Undrum, "A novel methodology for comparing $\mathrm{CO}_{2}$ capture options for natural gas-fired combined cycle plants," Adv. Environ. Res. 7(4), 901911 (2003).

${ }^{2}$ T. C. Lieuwen, V. McDonell, E. Petersen, and D. Santavicca, "Fuel flexibility influences on premixed combustor blowout, flashback, autoignition, and stability," J. Eng. Gas Turbines Power 130(1), 011506 (2008).

${ }^{3}$ M. Ni, D. Y. C. Leung, M. K. H. Leung, and K. Sumathy, "An overview of hydrogen production from biomass," Fuel Process. Technol. 87(5), 461-472 (2006).

${ }^{4}$ A. Gruber, J. H. Chen, D. Valiev, and C. K. Law, "Direct numerical simulation of premixed flame boundary layer flashback in turbulent channel flow," J. Fluid Mech. 709, 516-542 (2012).

${ }^{5}$ U. Ahmed, N. A. K. Doan, J. Lai, M. Klein, N. Chakraborty, and N. Swaminathan, "Multiscale analysis of head-on quenching premixed turbulent flames," Phys. Fluids 30(10), 105102 (2018).

${ }^{6}$ O. J. Ugarte, V. Bychkov, J. Sadek, D. Valiev, and V. y. Akkerman, "Critical role of blockage ratio for flame acceleration in channels with tightly spaced obstacles," Phys. Fluids 28(9), 093602 (2016).

${ }^{7}$ M. Boger, D. Veynante, H. Boughanem, and A. Trouvé, "Direct numerical simulation analysis of flame surface density concept for large eddy simulation of turbulent premixed combustion," Proc. Combust. Inst. 27(1), 917-925 (1998).

${ }^{8} \mathrm{R}$. Borghi and D. Dutoya, "On the scales of the fluctuations in turbulent combustion,” Symp. (Int.) Combust. 17(1), 235-244 (1979).

${ }^{9} \mathrm{~W}$. Kollmann and J. H. Chen, "Pocket formation and the flame surface density equation," Proc. Combust. Inst. 27(1), 927-934 (1998).

${ }^{10}$ S. B. Pope, "The evolution of surfaces in turbulence," Int. J. Eng. Sci. 26(5), 445469 (1988).

${ }^{11}$ S. M. Candel and T. J. Poinsot, "Flame stretch and the balance equation for the flame area," Combust. Sci. Technol. 70(1-3), 1-15 (1990).

${ }^{12} \mathrm{~N}$. Chakraborty and R. S. Cant, "Influence of Lewis number on curvature effects in turbulent premixed flame propagation in the thin reaction zones regime," Phys. Fluids 17(10), 105105 (2005).

${ }^{13}$ S. H. Kim and H. Pitsch, "Scalar gradient and small-scale structure in turbulent premixed combustion," Phys. Fluids 19(11), 115104 (2007).

${ }^{14}$ N. Chakraborty, E. R. Hawkes, J. H. Chen, and R. S. Cant, "The effects of strain rate and curvature on surface density function transport in turbulent premixed methane-air and hydrogen-air flames: A comparative study," Combust. Flame 154(1-2), 259-280 (2008).

${ }^{15}$ N. Chakraborty, M. Klein, D. Alwazzan, and H. G. Im, "Surface density function statistics in hydrogen-air flames for different turbulent premixed combustion regimes," Combust. Sci. Technol. 190(11), 1988-2002 (2018).

${ }^{16} \mathrm{U}$. Ahmed, R. Prosser, and A. J. Revell, "Towards the development of an evolution equation for flame turbulence interaction in premixed turbulent combustion," Flow, Turbul. Combust. 93(4), 637-663 (2014).

${ }^{17} \mathrm{~N}$. Chakraborty and N. Swaminathan, "Influence of the Damköhler number on turbulence-scalar interaction in premixed flames. I. Physical insight," Phys. Fluids 19(4), 045103 (2007).

${ }^{18}$ C. Dopazo, L. Cifuentes, J. Martin, and C. Jimenez, "Strain rates normal to approaching iso-scalar surfaces in a turbulent premixed flame," Combust. Flame 162(5), 1729-1736 (2015).

${ }^{19} \mathrm{C}$. Dopazo, L. Cifuentes, J. Hierro, and J. Martin, "Micro-scale mixing in turbulent constant density reacting flows and premixed combustion," Flow, Turbul. Combust. 96(2), 547-571 (2016).

${ }^{20}$ E. R. Hawkes and J. H. Chen, "Comparison of direct numerical simulation of lean premixed methane-air flames with strained laminar flame calculations," Combust. Flame 144(1-2), 112-125 (2006).
${ }^{21}$ R. Sankaran, E. R. Hawkes, J. H. Chen, T. Lu, and C. K. Law, "Structure of a spatially developing turbulent lean methane-air Bunsen flame," Proc. Combust. Inst. 31(1), 1291-1298 (2007).

${ }^{22}$ H. Wang, E. R. Hawkes, J. H. Chen, B. Zhou, Z. Li, and M. Aldén, "Direct numerical simulations of a high Karlovitz number laboratory premixed jet flameAn analysis of flame stretch and flame thickening," J. Fluid Mech. 815, 511-536 (2017).

${ }^{23}$ S. Chaudhuri, H. Kolla, H. L. Dave, E. R. Hawkes, J. H. Chen, and C. K. Law, "Flame thickness and conditional scalar dissipation rate in a premixed temporal turbulent reacting jet," Combust. Flame 184, 273-285 (2017).

${ }^{24}$ A. Sandeep, F. Proch, A. M. Kempf, and N. Chakraborty, "Statistics of strain rates and surface density function in a flame-resolved high-fidelity simulation of a turbulent premixed bluff body burner," Phys. Fluids 30(6), 065101 (2018).

${ }^{25}$ T. Kitano, T. Tsuji, R. Kurose, and S. Komori, "Effect of pressure oscillations on flashback characteristics in a turbulent channel flow," Energy Fuels 29(10), 68156822 (2015).

${ }^{26}$ U. Ahmed, A. L. Pillai, N. Chakraborty, and R. Kurose, "Statistical behavior of turbulent kinetic energy transport in boundary layer flashback of hydrogen-rich premixed combustion,” Phys. Rev. Fluids 4(10), 103201 (2019).

${ }^{27}$ A. L. Pillai and R. Kurose, "Combustion noise analysis of a turbulent spray flame using a hybrid DNS/APE-RF approach," Combust. Flame 200, 168-191 (2019).

${ }^{28} \mathrm{Y}$. Hu and R. Kurose, "Nonpremixed and premixed flamelets LES of partially premixed spray flames using a two-phase transport equation of progress variable," Combust. Flame 188, 227-242 (2018).

${ }^{29}$ U. Ahmed, C. Turquand d'Auzay, M. Muto, N. Chakraborty, and R. Kurose, "Statistics of reaction progress variable and mixture fraction gradients of a pulverised coal jet flame using direct numerical simulation data," Proc. Combust. Inst. 37(3), 2821-2830 (2019).

${ }^{30}$ T. Hara, M. Muto, T. Kitano, R. Kurose, and S. Komori, "Direct numerical simulation of a pulverized coal jet flame employing a global volatile matter reaction scheme based on detailed reaction mechanism," Combust. Flame 162(12), 4391-4407 (2015).

${ }^{31}$ C. Turquand d'Auzay, U. Ahmed, A. L. Pillai, N. Chakraborty, and R. Kurose, "Statistics of progress variable and mixture fraction gradients in an open turbulent jet spray flame," Fuel 247, 198-208 (2019).

${ }^{32} \mathrm{~J}$. A. Miller and C. T. Bowman, "Mechanism and modeling of nitrogen chemistry in combustion," Prog. Energy Combust. Sci. 15(4), 287-338 (1989).

${ }^{33}$ B. P. Leonard, "A stable and accurate convective modelling procedure based on quadratic upstream interpolation," Comput. Methods Appl. Mech. Eng. 19(1), 59-98 (1979).

${ }^{34}$ J. Lee, S. Yoon Jung, H. Jin Sung, and T. A. Zaki, "Effect of wall heating on turbulent boundary layers with temperature-dependent viscosity," J. Fluid Mech. 726, 196-225 (2013).

${ }^{35} \mathrm{H}$. Abe, H. Kawamura, and Y. Matsuo, "Surface heat-flux fluctuations in a turbulent channel flow up to $R e_{\tau}=1020$ with $P r=0.025$ and 0.71 ," Int. J. Heat Fluid Flow 25(3), 404-419 (2004).

${ }^{36} \mathrm{~V}$. Moureau, C. Bérat, and H. Pitsch, "An efficient semi-implicit compressible solver for large-eddy simulations," J. Comput. Phys. 226(2), 1256-1270 (2007).

${ }^{37}$ F. A. Williams, Combustion Theory, 2nd ed. (CRC Press, 1994).

${ }^{38}$ A. Ern and V. Giovangigli, Multicomponent Transport Algorithms (Springer Verlag, Heidelberg, 1994).

${ }^{39}$ R. Kee, F. Rupley, and J. Miller, "Chemkin-II, A FORTRAN chemical kinetics package for the analysis of gas-phase chemical kinetics," Technical report, Sandia National Laboratories, Albuquerque, NM, Livermore, CA, 1989.

${ }^{40} \mathrm{~T}$. J. Poinsot and S. K. Lelef, "Boundary conditions for direct simulations of compressible viscous flows," J. Comput. Phys. 101(1), 104-129 (1992).

${ }^{41}$ H. Pitsch, A C ++ Computer Program for 0D Combustion and 1D Laminar Flame Calculations, available at http://www.itv.rwth-aachen.de/en/downloads/ flamemaster/.

${ }^{42}$ R. D. Moser, J. Kim, and N. N. Mansour, "Direct numerical simulation of turbulent channel flow up to $\mathrm{Re}_{\tau}=590$," Phys. Fluids 11(4), 943-945 (1999). 
${ }^{43} \mathrm{~N}$. Chakraborty, "Comparison of displacement speed statistics of turbulent premixed flames in the regimes representing combustion in corrugated flamelets and thin reaction zones," Phys. Fluids 19(10), 105109 (2007).

${ }^{44} \mathrm{~T}$. Echekki and J. H. Chen, "Unsteady strain rate and curvature effects in turbulent premixed methane-air flames," Combust. Flame 106(1-2), 184-202 (1996).

${ }^{45}$ T. Echekki and J. H. Chen, "Analysis of the contribution of curvature to premixed flame propagation," Combust. Flame 118(1-2), 308-311 (1999).
${ }^{46} \mathrm{C}$. Dopazo and L. Cifuentes, "The physics of scalar gradients in turbulent premixed combustion and its relevance to modeling," Combust. Sci. Technol. 188(9), 1376-1397 (2016).

${ }^{47}$ R. Yu, T. Nillson, X.-S. Bai, and A. N. Lipatnikov, "Evolution of averaged local premixed flame thickness in a turbulent flow," Combust. Flame 207, 232-249 (2019).

${ }^{48}$ M. Klein, D. Alwazzan, and N. Chakraborty, "A direct numerical simulation analysis of pressure variation in turbulent premixed Bunsen burner flames-Part 1: Scalar gradient and strain rate statistics," Comput. Fluids 173, 178-188 (2018). 